\title{
Prediction of Electrolyte Conductivity: Results from a Generalized Molecular Model Based on Ion Solvation and a Chemical Physics Framework
}

\author{
Kevin L. Gering \\ Idaho National Laboratory (INL) \\ P.O. Box 1625, Idaho Falls, ID 83415-3732 \\ kevin.gering@inl.gov
}

\begin{abstract}
Ionic conductivity is a foremost transport property that is extensively used to characterize and screen electrolyte systems. Although bulk measurements are done on the macroscopic scale, electrolytic conductivity has its foundation on molecular-scale interactions between solvent and ionic species. Correct interpretations of these molecular interactions and related quantities enable a balanced, comprehensive understanding of conductivity behavior with respect to system conditions (solvent composition, salt concentration and temperature). This work introduces a new methodology that achieves accurate predictions of electrolyte conductivity for a wide range of conditions, based on molecular, physical, and chemical terms. The formalism is universal, making it valid for aqueous and non-aqueous systems alike. The immediate application of the resultant model is candidate electrolytes for lithium-ion and sodium-ion batteries, although many other applications abound for systems that utilize liquid electrolytes. Conductivity predictions are compared to experimental data for a number of electrolytes over a wide range of conditions, demonstrating that exceptional accuracy is attained because the robust model captures multiple salient contributions to conductivity behavior. Model accuracy is well maintained over multisolvent systems and for extended salt concentrations.
\end{abstract}

Keywords: electrolyte, ionic conductivity, ion solvation, chemical physics, lithium battery 


\begin{abstract}
Funding: This work was performed through support from the INL Laboratory Directed Research and Development (LDRD) program office and the U.S. DOE Vehicle Technologies Program Office.
\end{abstract}

\title{
1. Introduction
}

Without question, electrolytic conductivity is the chief transport property used to evaluate candidate electrolyte systems for a number of electrochemical energy storage devices (batteries, capacitors, etc.). Conductivity trends over electrolyte composition and temperature are a unique "thumbprint" of a given chemical system. However, conductivity can exhibit complex behavior that is often poorly understood or misunderstood, and thus was treated by empirical or semiempirical methods in early works [1,2]. Thus, a more rigorous, robust approach is needed to describe electrolytic conductivity that accounts for key contributions stemming from molecular scale interactions. This work addresses this need by considering key phenomena that influence conductivity: solvent-ion interactions (ion solvation) and solvated ion sizes, ion-ion interactions (ion association and electrostatic interactions), viscosity, counter-ion transport, ionic hopping and ionic random motion effects. Conductivity predictions are performed within a larger electrolytes modeling package known as the Advanced Electrolyte Model (AEM), a contemporary modeling tool developed under the US DOE Advanced Technology Development Program. One advantage of this modeling approach lies in its usage as an exploratory screening tool that can quickly determine optimal electrolyte compositions based on conductivity profiles or other parameters, as assessed over ranges of salt concentration, solvent composition, and temperature. Through the AEM, conductivity and other property predictions can now be made 
under numerous conditions for electrolytes that perhaps have not yet been studied or sparingly studied due to resource limitations. Within the larger modeling architecture developed by the author, conductivity is just one of many properties provided under a genomic-level investigation.

Herein the focus of the model predictions is battery electrolytes for Li-ion and Na-ion cells, although the modeling framework can be easily adapted to other non-battery applications. The systems of interest include solvents such as propylene carbonate (PC), ethylene carbonate (EC), mono-fluoro ethylene carbonate (FEC), ethyl methyl carbonate (EMC), dimethyl carbonate (DMC), diethyl carbonate (DEC), gamma butyrolactone (GBL), ethyl acetate (EA), 1,2 dimethoxyethane (DME), methyl butyrate (MB) and ethyl propionate (EP). Salts investigated include lithium hexafluorophosphate $\left(\mathrm{LiPF}_{6}\right)$, lithium bis(fluorosulfonyl)imide (LiFSI), lithium bis(trifluoromethanesulfonyl)imide (LiTFSI), and lithium bis(oxalate)borate (LiBOB), as well as some Na-ion counterparts to these salts. The AEM component library contains these and other compounds relevant to battery applications.

\section{Theoretical Basis}

\subsection{Overview of Approach}

The passage of current through an electrolyte liquid is facilitated by the movement of free ions within the liquid. However, "free ions" experience a number of constraining influences as they move throughout the electrolyte region, wherein the environment imposes interactive molecular effects (solvent-to-ion, ion-to-ion, and solvent-to-solvent) that ultimately determine 
the efficiency of transport for a particular set of positive and negative ions. In a theoretical sense, capturing the realities of conductivity behavior for electrolyte systems is an enormous challenge using a molecular-based modeling approach. Accurate concepts are those that target the foremost parameters that describe the various molecular-scale interactions and how they impact bulk macro-scale properties such as viscosity, conductivity, activity coefficients, etc.

Figure 1 shows the general approach developed by this work to predict solution conductivity, speaking to the complex interplay of parameters tied to this property. It is seen that ion solvation plays a seminal role in Fig. 1, emphasizing the importance of ion solvation phenomena in electrolyte behavior. Noteworthy influences on conductivity include solvent-ion interactions (sometimes termed as dielectric drag or friction), viscosity, ion association, counterion transport, random motion of ions, ion skipping (hopping) and other colligative effects. The modeling approach developed herein is universal in that it treats all electrolytes with the same set of governing equations, and hence, is unbiased toward the chemical system under investigation.

\subsection{Chemical Physics Framework}

Many important molecular processes involving battery electrolytes occur at spatial scales from 1-100 $\mathrm{nm}$ and timescales in the 1-100 $\mathrm{ns}$ range. Lithium desolvation and transport through surface-to-electrolyte interphase (SEI) films are examples of processes that can become rate limiting at particular conditions, such as at low temperatures or high cycling rates. In other cases, determination of macroscale properties (e.g., conductivity) by ab initio techniques may require an extensive sampling of molecular microstates to achieve adequate resolution of an average 
value at the chosen conditions. Added to this is the high variability that electrolyte regions can undergo during cell operations, such as in the double layer (DL) wherein properties change over the span of several seconds, minutes or hours according to the electrolyte concentration gradients that develop therein. In general, ab initio techniques have practical limits not capable of resolving dynamic molecular interactions well into the ns region, and they can't be economically used to render a comprehensive list of properties over broad range of conditions seen within the DL or for other optimization tasks. Thus, a more robust approach is required to gain information of meaningful fidelity at a fraction of the computing time of DFT/MD methods to enable sameday evaluation of perhaps thousands of conditions for systems of interest. Contemporary chemical physics (statistical-mechanical) models provide a powerful approach toward quantifying molecular-scale behavior in multi-component electrolytes and other chemical systems [3-13], and can provide highly detailed analysis on a number of key metrics pertinent to electrochemical systems.

The chemical physics or statistical-mechanical basis of the modeling approach herein is found in the non-primitive non-restricted associative form of the Mean Spherical Approximation (NPNRAMSA), a powerful computational approach to accurate interpretation of chemical physics behind real electrolyte systems. The NPNRAMSA enables robust predictions over solvent composition, salt concentration, temperature, and permittivity domains. Associative forms of MSA have been used with good success in predicting properties of non-ideal aqueous and non-aqueous electrolytes that undergo noteworthy ion association [9]. The NPNRAMSA form captures explicit information regarding solvated ion sizes and incorporates an exponential 
modification to MSA that corrects for inherent inadequacies of the original MSA theory [12-16], and allows for application of MSA to very high salt concentrations.

Overall, molecular scale interactions are considered within a framework that incorporates pertinent chemical, physical, and colligative contributions of the various species. Foremost initial parameters for NPNRAMSA include solvated ion sizes, solution densities, permittivity, temperature, ionic number densities, and thermodynamic governing equations covering ion association (e.g., ion pair formation) under equilibrium conditions. Effects from ion solvation are explicitly considered in terms of reference solvent residence times and average ion-solvent ligand distances, both of which influence the effective solvated ionic radii. Regarding the "nonprimitive" attribute of this modeling approach, the solution and solvent permittivity quantities are treated directly as colligative properties over salt concentration, wherein the change (decrease) of permittivity due to the presence of ions is explicitly derived and tied to Mass Action Law expressions for electrolyte species. Lastly, through NPNRAMSA the AEM estimates ion pair and ion triplet populations, as well as the onset of solid solvate formation. It is important to quantify these species because they can act as precursors to precipitated solids, and impact the effective ionic strength (and related conductivity) of an electrolyte solution. It should be noted that the NPNRAMSA as developed here is not limited to symmetric electrolytes (e.g., 1:1), but can be applied to any ionic stoichiometry in the native salt (1:1, 2:2, 1:2, 2:1, etc.). For example, the AEM has been successfully applied to electrolytes containing the salt $\mathrm{Li}_{2} \mathrm{~B}_{12} \mathrm{~F}_{12}$.

Chemical physics models such as the AEM are inherently very fast and efficient on a computational basis. This is due to time-averaged values of metrics for key molecular 
interactions that are assigned up front. For example, the AEM provides accurate rendering of a large set of diverse properties on the order of $\geq 10^{6}$ quicker than density functional theory (DFT) and molecular dynamics (MD) counterparts, and provides some macroscale properties typically out of reach of the ab initio techniques. With that said, NPNRAMSA is highly complementary with DFT and MD methods, for example, acting to confirm energy-minimized configurations of solvated ion configurations (DFT) or average solvent residence times around ions (MD).

\subsection{The Roles of Ion Solvation and Related Properties}

Ions in solution are known to experience solvation due to the attraction between their ionic charge and the opposite charge expressed by one or more solvent dipoles. This attraction results in a statistically significant concentration of near-neighbor solvent molecules around an ion, which comprise the solvation environment around that ion, sometimes referred to as a "coordination shell". The phenomenon of ion solvation has a central influence on thermodynamic, chemical and physical properties of electrolytes, including conductivity. Common metal cations such as $\mathrm{Na}^{+}, \mathrm{Li}^{+}, \mathrm{Mg}^{2+}$, etc. are generally more heavily solvated than anions because they are relatively small (and hence have a greater surface charge density) and because the negative dipole of many polar solvents is more sharply defined as compared to the positive dipole. Excellent summaries of topics pertaining to ion solvation can be found in the open literature [17-19]. Ion solvation is quantified within the AEM using two principal parameters: the average solvent residence time around ion $\mathrm{j}\left(\tau_{\mathrm{j}}\right)$, and the solvated ionic diameter $\left(\sigma_{\mathrm{j}}\right)$ for an arbitrary $\mathrm{j}$. 
A review of ion solvation size quantities is in order, which can be done by comparing lithium to potassium ions in water (see Figure 3 in reference 6). There are three basic types of ion diameters that are considered by the AEM: a bare ion diameter (superscript 'cr' herein), a hard sphere (HS) solvated diameter, and an "effective" (eff) solvated diameter. The bare ion size is simply evaluated through crystalline data or Pauling values that have been compiled in the literature $[17,20]$. The HS diameter is akin to a collision diameter, that is, the largest average size of solvated ion that can be assigned a rigid spherical structure at the prevailing molecular collision rate, solution density and other system-specific conditions. The effective diameter is representative of an effective transport size of an ion, such as that commonly referred to as a Stokes diameter. Effective diameters for cations and anions are generally larger than their HS counterparts. Note that HS and eff quantities each represent time-averaged values at conditions of interest, where HS diameters are used directly in the NPNRAMSA formalism and eff diameters are used within expressions related to transport quantities. As such, some of these dimensions account for partial or fractional solvent molecules. That is, in reality from a statistical perspective, average solvated ion dimensions might not be based on whole numbers of solvents.

The approximate magnitudes of the three diameter types have been gained from experimental data and other modeling techniques [e.g., 18,21]. Per Fig. 3 in [6], the higher charge density of the lithium ion results in a greater extent of solvation, as reflected in the magnitude of the lithium HS and eff diameters in comparison to its bare ion size. In contrast, the low charge density of the potassium ion promotes considerably less solvation in water. A related distinction that can be made regarding solvent structure around ions is that solvent "crowding" or densification is promoted by smaller ions due to their relatively high surface charge density. This 
aspect is reflected in higher energies of cation solvation for smaller cations in water [17]. Also

present is the "thawed" solvent region, which represents the fraction of the system volume that is occupied by solvent molecules whose solvent-solvent association has been disrupted by the presence of an ion, and yet which do not directly participate as ion solvators. This is a complementary concept to that of structure-breaking mechanisms in electrolyte viscosity [6]. An overlay of numerous "pictures" over time of a solvated ion in solution would yield composite average images for HS, eff, and thawed regions.

In general terms, solvated ion sizes are functions of the chosen solvent(s), ion concentrations, and temperature. Solvated radii tend to decrease as the salt concentration is increased due to greater competition among ions for solvent molecules at higher salt levels. And, solvated ion diameters increase at lower temperatures due to the lesser rate of molecular collisions that accompany lower thermal energies. Trends in ion solvation numbers $\left(\mathrm{n}_{\mathrm{s}}\right)$ over concentration and temperature mirror the trends of solvated ion sizes, decreasing over higher salt concentration and higher temperatures [22-26]. Li-ion electrolytes having common aprotic solvents have cation solvation numbers that usually exceed unity at modest salt concentrations (wherein $\mathrm{n}_{\mathrm{s}}$ values generally range from 2 to 5), and solvation numbers are not limited to integer values [18, 21-39]. Ion solvation shells represent solvent molecules that are able to position (coordinate) themselves around an ion due to electrostatic forces, over a statistically meaningful amount of time, and are not typically comprised of solvent species permanently bonded to an ion. It should be noted that while anions are generally much less solvated than cations in conventional battery electrolytes and aqueous systems, the extent of anion solvation is not 
negligible [e.g., 40-43], and it contributes to molecular and macroscopic properties of electrolytes such as viscosity [6] on which conductivity is highly dependent.

Another metric of ion solvation is the average solvent residence time around ion $\mathrm{j}$, denoted herein as $\tau_{\mathrm{j}}$. This term is a dynamic quantity representing the average duration of a solvent molecule in a coordination structure around arbitrary ion $\mathrm{j}$, and denotes relative stability of solvated ion structures. In general terms, magnitudes of residence times are greater among cation-solvent interactions (compared to anion-solvent), since many electrolyte solvents have one or more well-defined negative dipoles, but have a much weaker positive dipole expression. Estimates of $\tau_{\mathrm{j}}$ can be gotten through experimental methods $[18,44]$, quantum chemistry studies [24], and by correlation with solvatochromic properties [13]. Of particular note are the computational studies that investigate cation hydration, per Rode et al. and Helm and Merbach, which among other metrics discuss mean residence times of water molecules within first, second, and sometimes third solvation shells around cations, as well as solvation numbers within these shells [e.g., 45-50 and related references]. Further discussion on ion solvation metrics is given in a previous paper covering molecular phenomena that influence viscosity [6].

\section{Mathematical Development}

The following section provides development of the governing equations and related terms for the transport model of conductivity. Central to the mathematical and theoretical development is the utilization of chemical physics quantities, many of them in the molecular scale. Within the statistical mechanical purview, quantities like ion sizes (per their scales above), their equilibrium 
quantities over concentration, their charges, solvent attributes, solvent-ion interactions, temperature, system density and packing fraction, etc., all have meaningful influence on electrolyte properties such as conductivity. This basis has been maintained for the expressions derived below to achieve consistency with the NPNRAMSA approach. A list of symbols and their meanings is given toward the end of this manuscript.

\subsection{Using Stokes' Law as a Starting Point}

Stokes' Law has long been a means to approximate movement of small particles through frictional media, but it suffers inaccuracies when applied to concentrated electrolytes. In one sense the raw form of Stokes' Law is analogous to the ideal gas law, which can be amended by more rigorous treatment of terms to provide more accurate predictions over a greater range of conditions. From the raw form of Stokes' Law and the definition of ionic mobility we can derive an expression that approximates ionic conductance $\lambda_{\mathrm{j}}$ given only the ionic charge, liquid viscosity, and the representative size of an ion $\mathrm{j}$ :

$$
\lambda_{j}=\frac{2\left|z_{j}\right|}{\eta^{\circ} \sigma_{j}^{\circ}}\left(\frac{0.82 \times 10^{-8}}{1 \times 10^{-10}}\right) \quad \text { at } \infty-\text { dilution }
$$

where the terms in the brackets are conversion factors that have a collective value of 82.0 , and represent inputs of centipoise (cP) for viscosity and Angstroms for the ion diameter.

As shown, this simple form is suited only for conditions of infinite dilution or very low concentrations wherein the viscosity $\left(\eta^{\circ}\right)$ an ion diameter $\left(\sigma_{j}^{\circ}\right)$ terms do not appreciably vary. 
However, more sophistication is required at higher concentrations due to a host of non-idealities introduced as the ion populations increase in proportion to the solvent, which alters the thermodynamic attributes of the electrolyte. In this work, amendments to Stokes' Law are made that cover seven contributions to ionic conductivity: solvent-ion effects, ion association, counterion diffusion, random motion of ions, ionic hopping, viscosity as a function of salt concentration, and solvated ion size that varies with salt concentration. Each of these contributions is discussed below. The revised form of Stokes' Law for ionic conductance is expressed as:

$$
\lambda_{j}=\left\{f_{\text {solvent-ion } j}, f_{\alpha}, f_{j k}^{\text {bif }}, f_{\text {random }}, f_{\text {hop }}\right\} \cdot\left[82.0 * \frac{2\left|z_{j}\right|}{\eta(\mathrm{c}) \sigma_{j}^{\text {eff }}(\mathrm{c}, \mathrm{T})}\right] \text { at higher salt conc. }
$$

The $f$ terms are functions and factors defined below that account for the various contributions to conductivity as mentioned above, allowing Eq. 1 to be converted to a robust expression that is viable over large ranges of salt concentration and temperature. These terms are necessary to reveal the impact on conductivity from various species interactions over salt concentration, as well as the influence from thermal conditions on the transport environment. Within the AEM this form is applied to any electrolyte type, whether charge-symmetric or non-symmetric. For simplicity, the following three expressions are given for 1:1 systems:

Net conductance from single ions (SI):

$$
\Lambda_{S I}=\sum_{j=i}^{n} \lambda_{j, S I} \quad \text { for } n \text { SI types }
$$

Net conductance from triple ions (TI): 


$$
\Lambda_{T I}=\sum_{j=i}^{n} \lambda_{j, T I} \quad \text { for } n \text { TI types }
$$

Specific molar conductivity $\left(\mathrm{mScm}^{-1}\right)$ :

$$
\kappa=c_{M}\left[\Lambda_{S I}+\Lambda_{T I}\right]
$$

It is noted that for 1:1 systems ion pairs are charge neutral.

Using the preferred placement of terms, the final form of the conductance expression for $\lambda_{j}$ is

$$
\lambda_{j}=v_{j} \alpha_{j}\left(\begin{array}{l}
\left(1-f_{\text {hop }, j}\right)\left(f_{\text {random }, j}\left(\frac{1}{1+f_{\text {solvent-ion } j}}\right)\left(\frac{1}{1+f_{j k}^{\text {Diff }}}\right)\left(\frac{\theta}{\Gamma}\right)_{\substack{\text { aver } \\
\text { non-hop }}}\left[82.0 * \frac{2\left|z_{j}\right|}{\eta(\mathrm{c}) \sigma_{j}^{e f f}(\mathrm{c}, \mathrm{T})}\right]\right)^{-1} \\
+f_{\text {hop }, j}\left(f_{\text {random, } j}\left(\frac{1}{1+f_{\text {solvent-ion } j}}\right)\left(\frac{1}{1+f_{j k}^{\text {Diff }}}\right)\left(\frac{\theta}{\Gamma}\right)_{\substack{\text { ave, } \\
\text { hop }}}^{-1}\left[82.0 * \frac{2\left|z_{j}\right|}{\eta(\mathrm{c}) \sigma_{j}^{c r}}\right]\right)^{-1}
\end{array}\right)^{-1}
$$

wherein porosity $(\theta)$ and tortuosity $(\Gamma)$ terms have been included for hopping and non-hopping regions. Inclusion of such terms allows for the expression to be applied to composite electrolytes that might contain one or more porous media (including polymers) wherein the ions travel. For traditional liquid electrolytes we have $\theta=\Gamma=1$.

Note that all ionic species will have a representative $\lambda_{j}$ expression. For 1:1 electrolytes this will entail two single ion and two triple ion expressions, yet for non-symmetric salts the definition of charged species will vary with the ion stoichiometry. 


\subsection{Solvent-Ion Effects (ion solvation and dielectric friction)}

Solvent-ion interactions in electrolytes provide a retarding effect on ionic movement due to the attractive solvation energy between solvent and an ion, and due to solvent dipole reorientation and relaxation that occurs to solvent species in near proximity of an ion. These effects are greater at lower salt concentration since there are more solvent molecules per ion. Also, these contributions are in addition to those of the bulk viscosity, and are akin to dielectric drag or friction between solvent and solute mentioned by Zwanzig and Nee [51], more recently Kurnikova et al. [52] and others [53-71].

$$
f_{\text {solvent-ion } j}=y_{\text {elec }} \chi_{p s} \alpha_{1} \frac{1}{\varepsilon_{\text {soln }}(c)}\left(\frac{M_{p s} \rho_{2}^{\circ}}{M_{2} \rho_{p s}^{\circ}}\right)\left(\frac{\eta_{p s}^{\circ}}{\eta_{p s, r e f}^{\circ}}\right)\left(\frac{\sigma_{j}^{e f f^{3}}-\sigma_{j}^{c r^{3}}}{\sigma_{p s}^{3}}\right) \sum_{i=j}^{k} f_{i}^{*}
$$

where

$$
f_{i}^{*}=\left(\frac{v_{i} \tau_{i}}{v \tau_{\text {Debye }}}\right)\left(\frac{\sigma_{i}^{e f f^{3}}-\sigma_{i}^{H S^{3}}}{\sigma_{i}^{H S^{3}}-\sigma_{i}^{c r^{3}}}\right)\left(\frac{\sigma_{i}^{H S^{2}}-\sigma_{i}{ }^{c r^{2}}}{\sigma_{i}^{H S^{2}}}\right)\left(\frac{\sigma_{i}^{H S}-\sigma_{i}{ }^{c r}}{\sigma_{i}^{c r}}\right)
$$

and the Debye relaxation time is

$$
\tau_{\text {Debye }}=\left(\frac{3 V_{p s} \eta_{m i x} N_{A v}\left(1 \times 10^{-24}\right)}{R T}\right)
$$


Here $\chi_{p s}$ is the mole fraction of pseudo-solvent not bound in solvation shells, i.e., the free solvent, $y_{\text {elec }}$ is the packing fraction within the electrolyte at conditions of interest, $\varepsilon_{\text {soln }}$ is the colligative solution permittivity, and other terms have their usual meanings (some found below).

\subsection{Viscosity as $\mathbf{f}($ conc)}

Electrolyte viscosity has a foremost influence on conductivity, as is readily demonstrated by looking at conductivity curves for a single electrolyte system at different temperatures. At lower temperatures the locus of maximum for an isothermal conductivity curve occurs at lower solute concentrations. For many electrolyte systems, this consequence is largely due to increasing viscosity at lower temperatures, and due less to possible increases in ion association. Although viscosity is a bulk property, it roots are clearly in the molecular realm and thus provides a corresponding impedance to the migration of ions through their molecular environment, as elucidated from conductivity data. Viscosity as functions of salt concentration, solvent composition and temperature is accurately predicted by AEM through a method previously published that maintains the bases of molecular-scale interactions and chemical physics [6]. This permits accuracy of conductivity to extend to high concentrations, and is also meaningful for high-fidelity predictions of other terms such as ionic diffusivities. In particular, having reliable estimates for viscosity that span several orders of magnitude allows conditions to be investigated that might otherwise be problematic, such as higher salt concentrations at low temperatures.

\subsection{Solvated ion sizes as $\mathbf{f}($ conc)}




\subsection{Ion-ion Interactions (ion association)}

Ion association (IA) in electrolytes is a thermodynamic consequence and is a key parameter in electrolyte conductivity because it decreases the number of free ion charge carriers. Full treatment of IA effects is very complex and is performed by the AEM over each condition of salt concentration, solvent composition, and temperature. Choices for the salt(s) and solvent(s) are of paramount importance in IA behavior. IA evaluations are done for symmetric and asymmetric salts, alike, with determination made for equilibrium quantities of remaining free single ions, ion pairs, triple ions, and the onset of solid solvates. Coupled to this is a multi-level iterative scheme by which the true equilibrium solution and solvent permittivities are determined, since permittivity in an electrolyte drops as salt concentration increases due to dielectric depression 
and related terms $[9,72]$. Permittivity is a macroscopic or bulk property based on the aggregate molecular interactions of electrolyte species and their electronic structures. It is a relative measure of charge screening or electric polarization in electrolytes, and thus determines the effective electrostatic force at work between charged species/loci and solvent. The AEM determines the true field-attenuated permittivity at each condition of composition and temperature at which equilibrium speciation is determined. Herein, alpha terms represent IA quantities determined through the modeling approach, i.e., $\alpha_{1}$ is the fraction of salt that exists as free ions and $\alpha_{3}$ is the fraction that exists as triple ions. Thus, for $1: 1$ electrolytes $f_{\alpha}$ in Eq. 2 is simply the corresponding $\alpha_{1}$ or $\alpha_{3}$ depending on which charge carrier is being considered in Eq. 6. Examples of IA outputs are given below in relation to one of the electrolyte case studies. The unique treatment of IA by AEM is rigorous in terms of several mass action laws (MALs), the ligand population for each solvent, and colligative effects on permittivity (dielectric depression), and will be covered in a separate manuscript. The MAL framework is summarized as follows:

$$
\begin{aligned}
& \mathrm{A}+\mathrm{B} \stackrel{\mathrm{K}_{1}}{\rightleftarrows} \mathrm{AB}_{\mathrm{SSIP}} \stackrel{\mathrm{K}_{2}}{\rightleftarrows} \mathrm{AB}_{\mathrm{CIP}} \stackrel{\mathrm{K}_{3}}{\rightleftarrows} \mathrm{ABA} \text { and/or } \mathrm{BAB} \\
& \text { \{Free Ions\} } \quad\left\{\begin{array}{l}
\text { Solvent-Shared } \\
\text { Ion Pairs }
\end{array}\right\} \quad\left\{\begin{array}{l}
\text { Contact } \\
\text { Ion Pairs }
\end{array}\right\} \quad\{\text { Triple Ions }\} \\
& \Uparrow \mathrm{K}_{\mathrm{sol}} \\
& (\mathrm{A}-\mathrm{S}-\mathrm{B})_{\downarrow} \\
& \text { \{Solid Solvate\} } \\
& \left.v_{\mathrm{A}} \mathrm{A}+v_{\mathrm{B}} \mathrm{B}+v_{\mathrm{S}} \mathrm{S} \stackrel{\mathrm{K}_{\mathrm{sal}}}{\rightleftarrows}\left(\mathrm{A}_{v_{\mathrm{A}}}-\mathrm{S}_{v_{\mathrm{S}}}-\mathrm{B}_{v_{\mathrm{B}}}\right)_{\downarrow} \quad \text { \{stable or metastable solid }\right\} \\
& K_{\text {sol }}=\frac{a_{A-S-B}}{a_{A}^{v_{A}} a_{B}^{v_{B}} a_{S}^{v_{S}}}=\frac{C_{M} \alpha_{s o l} y_{A-S-B}}{\left(C_{M} y_{ \pm}\right)^{v}\left(v_{A} \alpha_{1, A}\right)^{v_{A}}\left(v_{B} \alpha_{1, B}\right)^{v_{B}} a_{S}^{v_{A} n_{S, A}}}
\end{aligned}
$$




\subsection{Counter-ion Diffusive Transport}

An ion $j$ undergoing Faradaic transport will encounter an obstructed path occupied in part by counter-ions and other non-j species. The presence of the counter-ion represents a fraction of volume unavailable for movement in by ion $j$. The relative extent of volume fraction between $j$ and non-j species will define the impeded path encountered by $j$. Accordingly, this "blocking action" by the counter-ion increases with increasing salt concentration and is heightened by larger counter-ion species. This phenomenon is mathematically described through the following expression that captures the effect of all counter-charged ions or neutral ion pairs on the migration of ion $j$, using a purely geometric basis. If we assign $\tilde{V}$ to represent the net molecular volume of a given specie at the concentration of interest, then

$$
f_{j k}^{D i f f}=\left(\alpha_{1} \sum_{i=j}^{k} \tilde{V}_{i}+\alpha_{3} \sum_{i=j}^{k} \tilde{V}_{T I_{i}}+\tilde{V}_{j k}\right)\left\{\frac{\alpha_{1} \tilde{V}_{k}+\alpha_{3} \tilde{V}_{k j k}+\tilde{V}_{j k}}{1-\left(\alpha_{1} \tilde{V}_{k}+\alpha_{3} \tilde{V}_{k j k}+\tilde{V}_{j k}\right)}\right\}
$$

A similar expression exists for $f_{k j}^{D i f f}$.

For diffusive effects on triple ions we have:

$f_{j k j}^{D i f f}=$ Same as RHS above, substituting $\alpha_{3}$ for $\alpha_{1}$ in the first factor.

$$
f_{k j k}^{\text {Diff }}=\text { (Similar approach) }
$$


In Eqs. (11) and (12) the volume effect of free solvent is not included since a portion of such solvent can become solvators and be co-transported with the Faradaic transport of ions, and since the impact of the molecular volume of free solvent is the same for all arbitrary $j$.

\subsection{Random Motion of Ions}

Electrolyte solutions are dynamic in the molecular realm. There is continuous movement and collisions between all species, the rate of which increases with increasing temperature. The result of this dynamic environment is an element of randomness to ionic movement (drunken sailor analogy), which has a direct impact on properties such as conductivity. The governing equation developed in this work is based on an assessment of those parameters that have an influence on either promoting or discouraging randomness of motion. Since only half the randomness in a conductive media is detrimental (wrong direction), the expression given herein correctly assigns a range of $1 / 2$ to unity for the randomness factor for arbitrary ion $\mathrm{j}, f_{\text {random }, j}$.

$$
\begin{aligned}
& f_{\text {random }, j}^{\prime}=\exp \left[-\left(\frac{1-y_{\text {elec }}}{y_{\text {elec }}}\right)\left(\frac{k T \tau_{\text {ref }}}{\frac{\pi}{6} \sigma_{j}^{H S^{3}} \eta_{\text {mix }}} \square 1 \times 10^{24}\right)\right. \\
& \text { I. II. } \\
& \text { • }\left(\frac{\alpha_{1} v_{j} c_{M} \sigma_{j}^{H S^{2}}}{\alpha_{1} c_{M}\left(v_{j} \sigma_{j}^{H S^{2}}+v_{i} \sigma_{i}^{H S^{2}}\right)+\left(1-\alpha_{1}\right) c_{M} \sigma_{I P}^{2}+\left[\frac{\rho_{m i x}-c_{M} M_{2}}{M_{P S}}-\sum_{i=j}^{k} n_{s, i}^{H S}\right] \sigma_{P S}^{2}}\right)
\end{aligned}
$$

III. 


$$
\text { - }\left(\frac{\dot{\sigma}^{c r}}{\dot{\sigma}^{c r}+\sigma_{p s}}\right)^{2}\left(1+\left(\frac{\sigma_{j}^{e f f^{3}}-\sigma_{j}^{c r^{3}}}{\sigma_{j}^{e f f^{3}}}\right)\left[\exp \left(-\tau_{j} / \tau_{r e f}\right)\right)\right]
$$

IV.

V.

Then

$$
f_{\text {random }, j}=1-\frac{1}{2}\left(1-f_{\text {random }, j}^{\prime}\right)
$$

Term I: $\quad$ Effect of electrolyte packaging fraction.

Term II: $\quad$ Thermal contribution; viscous contribution; absolute size ( $H S$ vol.) of species of interest.

Term III: $\quad$ Surface of $j$ relative to net surface of all species ("surface fraction"); $H S$ Basis; Range 0 to 1.

Term IV: $\quad$ Surface of bare "average" ion over surface of rotation composed of average bare ion and solvent (composite or coupled contribution from ions is provided by this average).

Term V: $\quad$ Relative solvation effects for ion $j$; range 1 to 2.

\subsection{Ionic Hopping}

Effective electrolyte conductivity is a statistical average of ionic conductance present under all likely solvation states viable for the given transport pathway. Various micro-states of ion solvation occur during Faradaic transport, from fully solvated to bare ion, due to local variations 
in electrolyte composition and to solvent exchange around ions as defined by finite solvent residence times (see Fig. 2). Ion hopping is then defined as a mode of ionic conductance under minimal or no solvation, resulting in a higher local ionic conductance. Specific to each ion type, ion hopping is mathematically defined here as the fraction (or, probability) of the ionic transport path that is travelled by an ion in its minimally solvated state. This aspect allows ion hopping to be another key selection and optimization tool for electrolytes since it represents the most efficient mode of Faradaic transport. The chemical physics approach under AEM is extended to predict occurrence of ionic hopping for both cations and anions, and how it impacts ionic conductance.

The expression developed for this work includes terms that cover relative rate, colligative and volume contributions. The resultant approach allows prediction of the probability that an ion will be encountering a minimally solvated state along its transport path.

$$
f_{\text {hop }, j}=x_{j, s} f_{\text {rate } j, s}\left(1-x_{s, j} f_{\text {vol } j, s}\right)
$$

where

$$
\begin{gathered}
x_{j, s}=\frac{\alpha_{1} v_{j} C_{\text {salt }}}{\alpha_{1} v_{j} C_{\text {salt }}+C_{\text {solvent }}} \\
x_{s, j}=1-x_{j, s} \\
f_{\text {rate } j, s}=\exp \left(-x_{s, j} \frac{\tau_{j}}{\tau_{\text {Debye }}}\right)
\end{gathered}
$$




$$
f_{\text {vol } j, s}=\left(\frac{r_{j}^{e f f^{3}}-r_{j}^{c r^{3}}}{\left(\left(\sigma_{\text {solvent }} f_{\text {shape }}+r_{j}^{c r}\right)^{3}-r_{j}^{c r^{3}}\right) y_{p: s, j}}\right)^{\frac{1}{3}}
$$

Here $\sigma_{\text {solvent }}$ is the equivalent spherical diameter of a solvent molecule and $f_{\text {shape }}$ is a shape factor that describes the solvent configuration in proximity to the chosen ion. The $1 / 3$ exponent on the $f_{v o l}$ term allows conversion to a one-dimensional translational ionic transport basis. The approximate packing fraction of solvent around ion $\mathrm{j}$ is given by $y_{p: s, j}$

Another consideration is to connect the ion hopping term for each ion to the rotational response of a solvent due to dipole-ion attraction. If the maximum extent of rotation is 180 degrees (based on the dipole position shifting from "completely away" to "completely toward" the ion), then we surmise that the extent of ion hopping is related to the actual angle of rotation $\theta_{\text {rotate }}$

$$
f_{\text {hop }, j} \propto 1-\frac{1}{2}\left(1-\cos \left(\theta_{\text {rotate }}\right)\right)
$$

That is, a solvent molecule that is able to completely realign toward an ion will be more likely to readily solvate that ion, thus preventing a minimally solvated state and the occurrence of ion hopping. On the other hand, small values of $\theta_{\text {rotate }}$ indicate a relatively irresponsive dipole shift toward the ion, and ion hopping is more likely. This type of assessment maintains relevance as other electrolyte systems are considered (gel, pseudo-polymer, polymer) and other conditions (low temperature) that rely more on ion hopping as a conductance mechanism [73]. 
For battery electrolytes this general approach finds utility in optimizing conventional liquid systems as well as polymeric and pseudo-polymeric electrolytes, in terms of finding formulations and their conditions that would promote ion hopping by the cation. Cationic transference numbers can be increased by finding electrolyte formulations that promote cation hopping and that contain anion receptors.

\section{Results}

Predictions of electrolyte conductivity were performed for systems with relevance to Li-ion batteries, since a wealth of laboratory data exists within this area. Calculations covered one or more parameters of salt concentration, solvent composition and temperature. The systems of interest include:

- $\quad \mathrm{PC}-\mathrm{LiPF}_{6}[74-76]$

- PC-EA-LiBOB [77]

- PC-DME-LiTFSI [78]

- EC-EMC-LiPF $6[79,80]$

- EC-EMC-LiBOB [81]

- PC-EC-DMC-LiPF 6 [82]

- EC-DEC-DMC-LiPF 6 [83]

- EC-DEC-DMC-EP-LiPF 6 [84]

- FEC-GBL-EMC-MB-LiFSI 
Figures 3-15 contain conductivity results for the above systems, comparing AEM predictions to lab measurements where available. The chemical physics of the AEM demonstrates robustness for single to multiple solvent systems, different types of solvents, different salts, and wide ranges of salt concentration and temperature. The overall average absolute percent deviation (AAPD) between AEM and lab data is low for the collective data (typically around five percent), with deviations for some datasets being less. Note that the symbol $\kappa$ is used in some of the figures to denote conductivity, a common use within other literature that covers this property. Two primary scales are used for salt concentration: Molar, M (mols $\mathrm{L}^{-1}$ solution) and molality, $\mathrm{m}$ (mols $\mathrm{kg}^{-1}$ solvent).

The AEM has served to detect errant lab data. For the case of PC-LIPF (Fig. 3) there is shown multiple sets of lab data presumably at the same conditions that have marked disagreement, as much as 15 percent within the region of 0.4 to 0.8 Molar salt concentration. This demonstrates that laboratory measurements, while completely necessary, can be impacted by varying conditions involving hardware calibration, skill of the lab technician, purity of samples, accuracy of temperature, etc. Hence, the AEM provides a consistent platform, immune to such variances, from which to assess key electrolyte properties such as conductivity.

Figure 4 shows the effective solvated ion diameters for the $\mathrm{PC}-\mathrm{LiPF}_{6}$ system, as functions of salt concentration and temperature. As explained above, the decrease in ion sizes is due to a diminished extent of ion solvation as greater salt content causes competition among ions for solvators. Diameters for solvated lithium are greater than those of $\mathrm{PF}_{6}^{-}$due to greater electrostatic attraction between the cation and the negative dipole of PC. Also, anion solvated sizes tend to 
change less over temperature and concentration (compared to lithium), since they are less solvated to begin with. Solvated ion diameter profiles are system dependent and component inter-dependent, and so will change with a different choice of electrolyte formulation. Note that since a chemical physics basis is employed, the solvated ion sizes represent molecular-scale time averages determined at each unique condition of salt concentration, solvent composition and temperature.

Mixed solvent systems involving PC are seen in Figs. 5 and 6, wherein PC-EA-LiBOB and PC-DME-LiTFSI are reported. The components EA and DME are added to lower system viscosity and improve conductivity, which must be done while avoiding undue ion association that can emerge if the lower-dielectric solvents such as EA and DME are in excess. The model predictions show very good agreement with lab data for these mixed-solvent systems.

Figure 7 contains lab data and modeling results for the EC-EMC-LiPF 6 electrolyte, a commonly studied system for Li-ion cells. Overall good agreement is seen between model and lab results, with AAPD being around five percent. Note that in Figs. 5-7 the AEM results go beyond the lab data in terms of salt concentration. This is done to allow for conditions within cells that represent concentration polarization. Having accurate conductivity values at high salt concentration enables better evaluations of double layer regions wherein there can be highly dynamic electrolyte gradients under battery operation. Figure 8 shows predictions of conductivity for the EC-EMC (3:7, wt.)- $\mathrm{LiPF}_{6}$ electrolyte for salt concentration to 3.5 molality, indicating a pronounced loss of conductivity at lower temperatures, which is due to high viscosity at these conditions [6]. 
Arrhenius behavior for conductivity of the EC-EMC (3:7, wt.)-LiPF 6 electrolyte is given in Fig. 9, using salt concentration as the parameter. Activation energies range from 10 to 36 $\mathrm{kJ} / \mathrm{mole}$, the higher value being for lower temperatures and the condition of 1.5 molality salt. Greater activation energies are anticipated at higher salt concentrations. The results of Fig. 9 are fundamentally important since concentration gradients will form within operating Li-ion cells, particularly at low temperatures, resulting in different local activation energies in conductivity.

Populations of ion association species are given in Fig. 10 for the EC-EMC-LiPF6 system at different solvent proportions, showing the summation of ion pairs ( $\left.\sum \mathrm{IP}\right)$ and triple ions $\left(\sum \mathrm{TI}\right)$. As anticipated, electrolyte richer in low-permittivity EMC shows a greater presence of all ion association species, which increases with increasing salt concentration. As mentioned above, locally higher salt concentrations will be manifest in Li-ion cells due to concentration polarization, and the instance of ion association will be promoted at these localities. For the 2:8 EC:EMC case, there is approximately $40 \%$ of the salt forming ion association species at 2 molality salt concentration.

Figure 11 shows AEM predictions for ionic hopping within the system EC-EMC (3:7, wt)$\mathrm{LiPF}_{6}$, investigating temperatures ranging from -30 to $60{ }^{\circ} \mathrm{C}$ and salt concentrations up to 3.5 molality. The results show that anions are much more prone toward ionic hopping, being minimally solvated, which helps to explain why anionic transference numbers for such electrolytes are generally above 0.5 by a significant amount. Also seen is that ionic hopping is statistically more likely to happen at high salt concentrations and higher viscosity conditions, 
such as at low temperatures. These results indicate that cationic transference numbers can be increased by having an anion receptor present in the electrolyte to provide association to anions. Yet, this benefit might come with the price of increasing viscosity due to the added structure in solution from anion-to-receptor interactions. Also, it is reasonable to ask whether the greater incidence of anion hopping for an EC-EMC based electrolyte would produce a problematic transport condition at the anode during discharge (build-up of anions near anode surface).

Conductivity for the EC-EMC (3:7, vol)-LiBOB system is given in Fig. 12. This system yields conductivity a little less than that of EC-EMC-LiPF , due to the $\mathrm{BOB}^{-}$anion being larger than $\mathrm{PF}_{6}{ }^{-}$. Yet, LiBOB-based electrolytes are desirable in one sense since they inhibit corrosion of aluminum current collectors.

A more rigorous test for any physics-based modeling approach is to consider more complex multi-component systems. Conductivity data and model predictions are compared in Figs. 13 and 14 for ternary solvent electrolytes EC-PC-DMC-LiPF 6 and EC-DEC-DMC-LiPF $6 . \quad$ In Fig. 15 a quaternary solvent system is investigated at temperatures down to $-60{ }^{\circ} \mathrm{C}$. Good predictive accuracy is maintained by the AEM in these more complex systems, covering both temperature and salt concentration. In fact, accuracy of the modeling method is well maintained regardless of the complexity encountered over the parameters of salt concentration, solvent composition, multi-solvent populations, and temperature. This verifies the robustness of the AEM.

The seven contributing factors to cationic conductivity are given in Fig. 16 for the FECGBL-EMC-MB (2:2:3:3, mass)-LiFSI system. Each contribution is plotted on a common scale 
to assess its relative influence on conductivity. It is seen that variance over salt concentration can be profound, as in the case of viscosity, solvent-ion effects, and ion association. Sensitivity to temperature is primarily seen in viscosity, random motion effects, and ion hopping effects. Note that the various curves in Fig. 16 are highly dependent on the choice of the electrolyte components, and that subtle changes in any curve will impact the resultant conductivity profiles that are obtained. Based on Fig. 16 we see that some contributions to electrolyte conductivity are minor. However, such proportion of impact will not always be the case, nor will viscosity effects always be of the magnitude shown in Fig. 16. For example, electrolytes that are comprised of low-viscosity low-permittivity solvents will undergo ion association that can outweigh viscosity effects as the salt concentration is raised. Also, systems with pseudo-polymer (gel) or polymer components will experience a significantly higher extent of ion hopping, compared to their liquid counterparts.

Figure 17 shows conductivity comparisons between $\mathrm{LiPF}_{6}$ and $\mathrm{NaPF}_{6}$ based electrolytes at $1 \mathrm{M}$ salt, wherein AEM was used to compare against lab data for the $\mathrm{NaPF}_{6}$ systems (data from Eric Dufek, personal communication). Per the preceding development, AEM accuracy has been established for electrolytes based on $\mathrm{LiPF}_{6}$. For the $\mathrm{NaPF}_{6}$ salt, the model results compare very favorably to the lab data, with only small deviations seen for the various solvent options; good agreement is also seen between AEM and Ponrouch et al. for such electrolytes [85]. A clear result from Fig. 17 is that Na-ion systems have the advantage of higher conductivity in most cases, which originates in the slightly lesser solvated environment of sodium ions due to their lower surface charge density compared to lithium. This decrease in cation solvation results in 
lowered solution structure and yields a corresponding decrease in viscosity [6], thereby improving conductivity.

Lastly, we consider optimization of multi-solvent electrolytes through Large-Scale Simulations (LSS). It is a proven concept that Li-ion electrolytes benefit in many ways from utilizing multiple solvents. Custom mixtures can be formulated that provide advantages such as lowering viscosity, inhibiting ion association, improving safety performance, and optimizing electrode surface films (SEI). However, it is no small task in the laboratory to determine which solvent proportions are optimal in a multi-solvent system for a given context, such as electrolytic conductivity. Toward this challenge, the AEM was designed to perform LSS that investigate numerous solvent proportions in mixed solvent electrolytes (many thousands to millions) to determine which compositions yield optimized performance according to one or more optimization criteria. LSS optimization results at low temperatures are of particular importance. The LSS optimization parameter can be conductivity, the product of conductivity and cation transference number, some measure of lithium desolvation that connects to the charge transfer process, the electrolyte diffusion coefficient, or any other such meaningful transport or thermodynamic quantity.

In Fig. 18 a simple case is considered for a three-solvent system, XYZ-EMC-GBL-LiPF, where $\mathrm{XYZ}$ is a proprietary solvent. The objective is to optimize the proportions of solvents over salt concentration and temperature, using conductivity as the optimization metric. Around 40,000 distinct conditions of $\left(\mathrm{C}_{\text {salt }}, \mathrm{x}_{\text {solvent }}, \mathrm{T}\right)$ were investigated during the LSS, wherein AEM calculated a full suite of properties at each condition. At low temperatures it is seen that more 
EMC is required (50-60\%) to provide superior conductivity, while GBL is seen to decrease in general over salt concentration due to viscosity effects. As temperature increases, solvent permittivity decreases and ion association becomes more widespread. As a consequence, GBL content needs to increase to improve permittivity because EMC and proprietary XYZ have relatively low dielectric constants. A minimum of 25 weight percent has been assigned to XYZ, and its presence is seen to improve conductivity at lower temperature and higher salt content. From Fig. 18 LSS results, an electrolyte can be designed that targets the desired range of salt concentration and temperature for the intended battery application. In general, as AEM LSS is applied to more complicated four or five solvent systems, perhaps with two salts, an enormous benefit arises in saving laboratory resources and accelerating the detection of optimal electrolyte formulations.

\section{Discussion}

Trends and magnitudes of conductivity are well predicted by the AEM for several electrolytes, considering wide ranges of solvent composition, salt concentration, and temperature. Conductivity maxima over salt concentration are predicted by the AEM with very good accuracy in most cases. The trends of such maxima with temperature are well captured by the AEM, and are the result of the combined chemical physics contributions in the previous development, and not due to an empirical correlation such as the Casteel-Amis equation [e.g., 86]. Also, the NPNRAMSA approach developed by the author allows consideration of high salt concentrations, in some cases approaching 4 molal salt for battery electrolytes, and higher in double-layer calculations (in application to aqueous systems the model can facilitate salt 
concentrations approaching 20 molal). Thus, the predictive accuracy of the model is achieved over a broad landscape of conditions. This capability underscores that the AEM is able to rigorously interpret the chemical physics effects of the molecular-scale electrolyte environment on ionic conductance and related properties. From Fig. 1, this ability is largely due to the keen interpretation of ion solvation effects that the model provides.

In some cases there is disagreement between published laboratory data, as was demonstrated with a key PC-based system (Fig. 3). This situation is compounded by the fact that some investigators who publish conductivity data do not include error bars, accurate temperature conditions ("RT" might vary within the room over time, or vary between investigators), or other relevant information regarding the integrity of their data. Yet, the accuracy of the AEM is generally well within the bounds established by reliable data from different sources, showing average absolute deviations of around 5\% for many conductivity data sets, and often less.

The computational method given herein lends itself to diagnostic analysis of factors that influence conductivity. These various and diverse contributions to conductivity will manifest more or less depending on the magnitude of salt concentration, solvent attributes, temperature, etc. Of the factors shown in Eq. (6), viscosity has the most influence, particularly at low temperatures and high salt concentrations. It is also worthwhile to consider conditions that impact local electrolyte behavior, such as in double layers, wherein the properties will shift during battery cycling according to the local environment and battery operational metrics. Selecting an optimal electrolyte formulation based on conductivity is therefore a complicated process of weighing contributions to conductivity against anticipated use conditions in the 
battery application. For example, selection of solvents and salt will be motivated by lowering viscosity, increasing cation transference, increasing ionic hopping, reducing the solvated cation size, while keeping ion association to an acceptable minimum. The methodology presented herein allows viewing such variables in concert.

The electrolytes investigated herein for $\mathrm{Li}$-ion and Na-ion cells constitute a small yet representative fraction of what has and is being investigated. The ever-growing pace of research worldwide calls for enabling tools such as AEM that will enhance the rate of technology development. One important aspect to the modeling approach is that a vastly high number of off-matrix conditions can be investigated to complement lab data, making the case that chemical physics methods can promote economic use of lab resources, accelerate attainment of knowledge, and broaden the foundation of information to capture a greater application space.

\section{Conclusions}

Chemical physics models having their roots in integral equation theory serve an essential role in simulating the real environment of complex electrolyte systems for batteries, and render results with highly economical use of computing resources. Such models are not intended to replace $a b$ initio techniques, but rather provide a logical complement. When combined, these tools offer outstanding synergy toward full characterization of electrolyte systems under an enormous variety of conditions. 
Robustness of the AEM has been confirmed over multiple electrolyte systems and numerous conditions relevant to Li-ion and Na-ion electrochemical cells. High predictive accuracy is well maintained by the AEM in these complex non-ideal systems, covering conductivity predictions over the parameters of salt concentration, solvent composition, multi-solvent populations, and temperature. This capability is made possible in large part due to the model's correct interpretation of ion solvation over wide conditions, a foundational attribute that underlies many aspects of electrolyte conductivity. The speed and accuracy of the modeling approach allows tremendous benefit toward accelerating electrolyte research and broadening the foundation of knowledge for these systems, and thus supports timely genomic-level investigations therein. While the generalized framework within the AEM has been applied here to battery electrolytes, it can be applied to many other non-battery targets.

\section{Acknowledgements}

The author expresses gratitude for the programmatic support provided by the INL Laboratory Directed Research and Development (LDRD) program and the U.S. DOE Vehicle Technologies Program Office. This work was performed under DOE-ID contract No. DE-AC0705ID14517. The author also thanks his colleagues from INL: Dr. Eric Dufek is acknowledged for providing conductivity data for Na-ion systems, and Dr. Joshua McNally is acknowledged for providing solvent-ion configurational data for Li-ion and Na-ion systems derived from DFT studies. 


\begin{tabular}{|c|l|l|}
\hline Symbol & Meaning & Units \\
\hline
\end{tabular}

\section{Greek Symbols:}

\begin{tabular}{|c|c|c|}
\hline$\alpha$ & extent of salt dissociation & -- \\
\hline$\varepsilon$ & $\begin{array}{l}\text { relative permittivity, } \\
\text { colligative basis }\end{array}$ & -- \\
\hline$\eta$ & viscosity & $\mathrm{cP}^{*}$ \\
\hline$\theta$ & porosity & -- \\
\hline$\theta_{\text {rotate }}$ & $\begin{array}{l}\text { angle of rotational } \\
\text { response of a solvent due } \\
\text { to dipole-ion attraction }\end{array}$ & degrees \\
\hline$\kappa$ & $\begin{array}{l}\text { specific molar } \\
\text { conductivity }\end{array}$ & $\begin{array}{l}\mathrm{mS} \mathrm{cm}-1 \text { or } \\
\mathrm{mOhm}^{-1} \mathrm{~cm}^{-1}\end{array}$ \\
\hline$\lambda_{\mathrm{j}}, \Lambda$ & $\begin{array}{l}\text { ionic and total } \\
\text { conductance }\end{array}$ & $\mathrm{m}^{2} \mathrm{~S} \mathrm{~mol}^{-1}$ \\
\hline$v$ & stoichiometric coefficient & -- \\
\hline$\sigma$ & ionic diameter & $10^{-10} \mathrm{~m}(\AA)$ \\
\hline$\tau$ & solvent residence time & $10^{-9} \mathrm{~s}$ \\
\hline$\tau_{\text {Debye }}$ & Debye relaxation time & $10^{-9} \mathrm{~s}$ \\
\hline$\chi$ & mole fraction & -- \\
\hline$\Gamma$ & tortuosity & -- \\
\hline \multicolumn{3}{|c|}{ English Symbols: } \\
\hline a & activity & -- \\
\hline$c$ or $C$ & concentration & Molar \\
\hline$f$ & function index & -- \\
\hline$k$ & Boltzmann's constant & $1.38065 \times 10^{-23} \mathrm{JK}^{-1}$ \\
\hline$K$ & equilibrium constant & (various) \\
\hline $\mathrm{m}$ & molality & mol kg ${ }^{-1}$ solvent \\
\hline M & Molar & mol L ${ }^{-1}$ solution \\
\hline $\mathrm{N}_{\mathrm{AV}}$ & Avogadro's Number & $6.022 \times 10^{23} \mathrm{~mol}^{-1}$ \\
\hline $\mathrm{r}$ & ionic radius & $10^{-10} \mathrm{~m}(\AA)$ \\
\hline $\mathrm{R}$ & gas constant & $8.3144 \mathrm{~J} \mathrm{~mol}^{-1} \mathrm{~K}^{-1}$ \\
\hline $\mathrm{T}$ & temperature & Kelvin \\
\hline
\end{tabular}




\begin{tabular}{|c|c|c|}
\hline $\mathrm{V}$ or $\tilde{\mathrm{V}}$ & molecular volume & $\begin{array}{l}\mathrm{m}^{3} ; \text { depends on } \\
\text { form of equation }\end{array}$ \\
\hline $\mathrm{x}$ & mole fraction & -- \\
\hline $\mathrm{y}$ & packing fraction & -- \\
\hline $\mathrm{y}_{ \pm}$ & mean activity coefficient & -- \\
\hline $\mathrm{z}_{\mathrm{j}}$ & ionic charge & -- \\
\hline \multicolumn{3}{|c|}{ Subscripts: } \\
\hline 1 & single ions & -- \\
\hline 2 & salt & -- \\
\hline 3 & triple ions & -- \\
\hline CIP & contact ion pair & -- \\
\hline elec & electrolyte & -- \\
\hline hop & ion hopping & -- \\
\hline IP & ion pair & -- \\
\hline$j, k$ & ion indices & -- \\
\hline $\operatorname{mix}$ & solvent mixture & -- \\
\hline PS & pseudo solvent & -- \\
\hline SI & single ion & -- \\
\hline soln & solution & -- \\
\hline SSIP & solvent-separated ion pair & -- \\
\hline TI & triple ion & -- \\
\hline \multicolumn{3}{|c|}{ Superscripts: } \\
\hline $\mathrm{cr}$ & crystalline or bare ion & -- \\
\hline Diff & diffusive & -- \\
\hline eff & effective & -- \\
\hline HS & hard sphere & -- \\
\hline o & salt-free & -- \\
\hline
\end{tabular}

$* 1 \mathrm{~kg} \mathrm{~m}^{-1} \mathrm{~s}^{-1}=1000$ centipoise 


\section{References}

[1] H. S. Harned and B. B. Owen, The Physical Chemistry of Electrolyte Solutions, $3^{\text {rd }}$ Ed., Reinhold Publishing Corporation, New York, 1958.

[2] R. A. Robinson and R. H. Stokes, Electrolyte Solutions, Butterworths Scientific Publications, London, 1955.

[3] L. L. Lee, Molecular Thermodynamics of Nonideal Fluids, Butterworth-Heinemann, Boston, 1988.

[4] Marshall, B. D. and W. G. Chapman, J. Chem. Phys. 139 (21) (2013) 214106.

[5] Keshawa P. Shukla and Walter G. Chapman, Mol. Phys. 91 (1997) 1075.

[6] K. L. Gering, Electrochim. Acta, 51 (2006) 3125.

[7] H. Zhao, M. C. dos Ramos, and C. McCabe, J. Chem. Phys. 126 (2007) 244503.

[8] W-B Liu, Y-G Li and J-F Lu, Ind. Eng. Chem. Res. 37 (1998) 4183.

[9] J. M. G. Barthel, H. Krienke, and W. Kunz, Physical Chemistry of Electrolyte Soutions, Modern Aspects, Steinkopff-Springer, Darmstadt, 1998.

[10] L. L. Lee, M. Llano-Restrepo, W. G. Chapman, and K. P. Shukla, J. Chin. I. Ch. E. 27 (4) (1996) 213.

[11] W.G. Chapman, K.E. Gubbins, G. Jackson, and M. Radosz, Fluid Phase Equilibria, 52 (1989) 31.

[12] L. H. Landis, Jr., Mixed Salt Electrolyte Solutions: Accurate Correlation for Osmotic Coefficients Based on Molecular Distribution Functions, Doctoral Dissertation, University of Oklahoma, 1985.

[13] K. L. Gering, A Molecular Approach to Electrolyte Solutions: Predicting Phase Behavior and Thermodynamic Properties of Single and Binary-Solvent Systems, Doctoral Dissertation, University of Oklahoma, 1989.

[14] H. C. Anderson and D. Chandler, J. Chem. Phys. 53 (1970) 547.

[15] D. Chandler and H. C. Anderson, J. Chem. Phys. 54 (1971) 26.

[16] H. C. Anderson and D. Chandler, J. Chem. Phys. 55 (1971) 1497.

[17] Y. Marcus, Ion Properties, Marcel Dekker, Inc., New York, 1997.

[18] Y. Marcus, Ion Solvation, John Wiley \& Sons, Chichester, 1985.

[19] A. Ben-Naim, Solvation Thermodynamics, Plenum Press, New York, 1987.

[20] L. Pauling, General Chemistry, $3^{\text {rd }}$ Ed., W. H. Freeman and Company, San Francisco, 1970.

[21] G. Licheri, G. Piccaluga, and G. Pinna, Chem. Phys. Lett. 35 (1975) 119.

[22] M. Morita, Y. Asai, N. Yoshimoto and M. Ishikawa, J. Chem. Soc., Faraday Trans. 94 (1998) 3451.

[23] S. Hyodo and K. Okabayashi, Electrochimica Acta 34 (11) (1989) 1551.

[24] O. Borodin and G. D. Smith, J. Phys. Chem. B 113 (2009) 1763.

[25] S-D. Han, O. Borodin, D. M. Seo, Z-B. Zhou and W. A. Henderson, J. Electrochem. Soc. 161 (14) (2014) A2042.

[26] Y. Marcus, J. Phys. Chem. B 118 (2014) 10471.

[27] B. Klassen, R. Aroca, M. Nazri, and G. A., Nazri, J. Phys. Chem. B 102 (1998) 4795.

[28] Y. Matsuda, H. Nakashima, M. Morita, Y. Takasu, J. Electrochem. Soc. 128 (12) (1981) 2552. 
[29] Z. Wang, B. Huang, R. Xue, L. Chen, and X. Huang, J. Electrochem. Soc. 145 (10) (1998) 3346.

[30] M. Morita, Y. Asai, N. Yoshimoto, and M. Ishikawa, J. Chem. Soc. Faraday Trans. 94 (1998) 3451.

[31] J. M. Alía and H. G. M. Edwards, Vibrational Spectroscopy 24 (2000) 185.

[32] S. Yanase and T. Oi, J. Nuc. Sci. and Tech. 39 (10) (2002) 1060.

[33] S. Hyodo and K. Okabayashi, Electrochemica Acta 34 (11) (1989) 1557.

[34] J. Barthel, R. Buchner, and E. Wismeth, J. Soln. Chem. 29 (10) (2000) 937.

[35] Z. Deng and D. E. Irish, J. Chem. Soc. Faraday Trans. 88 (19) (1992) 2891.

[36] Z. Deng and D. E. Irish, Can. J. Chem. 69 (11) (1991) 1766.

[37] R. Buchner and G. Hefter, J. Sol. Chem. 31 (7) (2002) 521.

[38] R. J. Blint, J. Electrochem. Soc. 142 (3) (1995) 696.

[39] S. Kobayashi et al., Proceedings of the Electrochemical Society 204th Meeting, Orlando, FL October 12-16, 2003.

[40] N. Peruzzi, P. Lo Nostro, B. W. Ninham and P. Baglioni, J. Soln. Chem. 44 (2015) 1224.

[41] J.N.C. Lopes and A.A.H. Padua, J. Phys. Chem. B 110 (2006) 3330.

[42] J.N.C. Lopes, M.F.C. Gomes and A.A.H. Padua, J. Phys. Chem. B 110 (2006) 16816.

[43] R. Jan, G. M. Rather and M. A. Bhat, J. Soln. Chem. 43 (2014) 685.

[44] A. I. Mishastin, Zh. Fiz. Khim. 55 (1981) 1507.

[45] C. F. Schwenk and B. M. Rode, Pure Appl. Chem. 76 (1) (2004) 37.

[46] L. R. Canaval, A. K.H. Weiss, B. M. Rode, Comp. and Theor. Chem. 1022 (2013) 94.

[47] S. Durdagi., T. S. Hofer, B. R. Randolf, B. M. Rode, Chem. Phys. Lett. 406 (2005) 20.

[48] T. S. Hofer, H. T. Tran, C. F. Schwenk, B. M. Rode, J. of Comp. Chem. 25 (2) (2003) 211.

[49] L. Helm and A. E. Merbach, Coord. Chem. Rev. 187 (1999) 151.

[50] Th. Kowall, F. Foglia, L. Helm, and A. E. Merbach, J. Am. Chem. Soc. 117 (1995) 3190.

[51] T. W. Nee and R. Zwanzig, J. Chem. Phys. 52 (1970) 6353.

[52] M. G. Kurnikova, D. H. Waldeck, and R. D. Coalson, J. Chem. Phys. 105 (2) (1996) 628.

[53] B. U. Felderhof, Molecular Phys. 49 (2) (1983) 449.

[54] G. van der Zwan and J. T. Hynes, J. Chem. Phys. 76 (1982) 2993.

[55] C. Millot, J. L. Rivail, R. Diquet, Chem. Phys. Lett. 162, (1989) 222.

[56] A. Chandra and B. Bagchi, J. Phys. Chem. 94 (1991) 8367.

[57] D. Kivelson and T. Keyes, J. Chem. Phys. 57 (1972) 4599.

[58] B. Bagchi and A. Chandra, in Advances in Chemical Physics, Vol. LXXX, edited by I. Prigogine and S. A. Rice (Wiley, New York, 1991), p. 247.

[59] M. Maroncelli, J. Mol. Liq. 57 (1993) 1.

[60] A. Chandra and B. Bagchi, J. Phys. Chem. 94 (1991) 3107.

[61] R. S. Hartman and D. H. Waldeck, J. Phys. Chem. 98 (1994) 1386.

[62] D. S. Alavi and D. H. Waldeck, J. Chem. Phys. 98 (1993) 3580.

[63] R. S. Hartman, D. S. Alavi, and D. H. Waldeck, Isr. J. Chem. 33 (1993) 157.

[64] D. S. Alavi, R. S. Hartman, and D. H. Waldeck, J. Chem. Phys. 98 (1993) 3585.

[65] R. S. Hartman, W. Konitsky, and D. H. Waldeck, J. Am. Chem. Soc. 115 (1993) 9692.

[66] A. Papazyan and M. Maroncelli, J. Phys. Chem. 95 (1991) 9219.

[67] M. Maroncelli, J. Phys. Chem. 94 (1991) 2084.

[68] P. G. Wolynes, Annu. Rev. Phys. Chem. 31 (1985) 345.

[69] M. Bruehl and J. T. Hynes, J. Chem. Phys. 96 (1992) 4068.

[70] D. S. Alavi, R. S. Hartman, and D. H. Waldeck, J. Chem. Phys. 92 (1990) 4055. 
[71] L. A. Philips, S. P. Webb, and J. H. Clark J. Chem. Phys. 83 (1985) 5810.

[72] G. Power, J. K. Vij and G. P. Johari, J. Phys. Chem. B 111 (2007) 11201.

[73] Y. Wang, B. Li, J. Ji and W-H Zhong, J. Power Sources 247 (2014) 452.

[74] J. Barthel, H. J. Gores and G. Schmeer, Ber. Bunsenges. Phys. Chem 83 (1979) 911.

[75] K. Kondo et al., J. Phys. Chem. B, 104 (2000) 5040.

[76] A. M. Christie and C. A. Vincent, J. Phys. Chem., 100 (1996) 4618.

[77] M. S. Ding and T. R. Jow, $204^{\text {th }}$ meeting of the Electrochemical Society, Abstract 299 (2003).

[78] A. Webber, J. Electrochem. Soc. 138 (9) (1991) 2586.

[79] M. S. Ding, K. Xu, S. S. Zhang, K. Amine, G. L. Henriksen, J. Electrochem. Soc. 148 (10), (2001) A1196.

[80] A. Nyman, M. Behm and G. Lindbergh, Electrochim. Acta 53 (2008) 6356.

[81] T. Fujii, M. Takehara and M. Ue, $202^{\text {nd }}$ meeting of the Electrochemical Society, Abstract 203 (2002).

[82] L. O. Valoen and J. N. Reimers, J. Electrochem. Soc. 152 (5), A882 (2005).

[83] Merck KGaA, Battery Materials (materials for energy storage devices), Darmstadt, Germany (March 2001).

[84] M. C. Smart, B. V. Ratnakumar and S. Surampudi, J. Electrochem. Soc. 149 (4) (2002) A361.

[85] A. Ponrouch, E. Marchante, M. Courty, J-M. Tarascon and M. R. Palacin, Energy Environ. Sci. 5 (2012) 8572.

[86] L. Werblan and A. Balkowska, J. Electroanal. Chem. 354 (1993) 25. 
Fig. 1: Relationship of contributing factors to ionic conductivity, as incorporated into the AEM modeling approach. Ion solvation is a foundational attribute that underlies many aspects of electrolyte conductivity.

Fig. 2: Conceptual rendering of ion hopping in a liquid electrolyte system. Various solvation microstates are shown as the ion moves from left to right under Faradaic transport.

Fig. 3: Conductivity data and model results for the $\mathrm{PC}-\mathrm{LiPF}_{6}$ system at $25^{\circ} \mathrm{C}$ as a function of salt concentration. Some non-trivial discrepancies are seen between the lab data [74-76]. AEM gives good fidelity overall, even past $3 \mathrm{M}$ salt.

Fig. 4: Effective (transport) solvated ion sizes determined by the AEM for the PC-LiPF 6 systems at -30 and $30{ }^{\circ} \mathrm{C}$. Solvated cation and anion sizes are seen to decrease with increasing salt concentration and with increasing temperature. In this system, solvated anion sizes are less than their cation counterparts.

Fig. 5: Conductivity data and model results for the PC-EA-LiBOB system at various temperatures and as a function of salt concentration. Lab data (dark circles, [77]) is compared to model results (solid curves).

Fig. 6: Conductivity data and model results for the PC-DME-LiTFSI system at $25{ }^{\circ} \mathrm{C}$ as a function of salt concentration. Lab data (dark circles, [78]) is compared to model results (solid curve). The slight bend in the curve at around 2.5 pseudo-molarity is due to model adjustments made in response to a critical packing fraction reached at that condition.

Fig. 7: Conductivity data and model results for the EC-EMC-LiPF 6 system at various temperatures and as a function of salt concentration. Lab data (dark circles, [79]) is compared to model results (solid curves). Overall average deviation between model and lab data is $5 \%$.

Fig. 8: AEM results for the EC-EMC-LiPF 6 system at various temperatures and as a function of extended salt concentration. The very slight bend in the curves at higher molality is due to model adjustments made in response to a critical packing fraction reached at those conditions, which is a function of temperature.

Fig. 9: Arrhenius analyses of conductivity based on AEM results for the EC-EMC-LiPF 6 system, using salt concentration (molality) as the parameter. Activation energies based on conductivity increase at lower temperatures and higher salt concentrations. This has implications for polarized Li-ion cells operating at low temperatures.

Fig. 10: Prediction of ion association species for the EC-EMC-LiPF 6 system at $30{ }^{\circ} \mathrm{C}$, as functions of solvent composition and salt concentration. The sum of ion pairs $\left(\sum \mathrm{IP}\right)$ is 
comprised of contact ion pairs, solvent-separated ion pairs and solvent-shared ion pairs, while the triple ion summation $\left(\sum \mathrm{TI}\right)$ is comprised of $\mathrm{ABA}^{+}$and $\mathrm{BAB}^{-}$species.

Fig. 11: Probability of ion hopping for $\mathrm{Li}^{+}$and $\mathrm{PF}_{6}{ }^{-}$in EC-EMC. Ion hopping is more prevalent for lesser-solvated ions (here, anions) and at lower temperatures.

Fig. 12: Conductivity data [81] and model results for the EC-EMC-LiBOB system at various temperatures and at $0.85 \mathrm{M}$ salt. Lab data (dark circles) is compared to model results (solid curve).

Fig. 13: Conductivity ( $\kappa)$ data [82] and model results for the EC-PC-DMC (27:10:63, vol.)$\mathrm{LiPF}_{6}$ system at various temperatures and over salt concentration. Lab data (symbols) is compared to model results (solid curves).

Fig. 14: Conductivity data [83] and model results for the EC-DEC-DMC-LiPF 6 system at 0.95 molality salt, two sets of solvent proportions and various temperatures. Lab data (dark circles) is compared to model results (solid curves). Good fidelity of model predictions is seen over the entire temperature range of -40 to $80{ }^{\circ} \mathrm{C}$.

Fig. 15: Conductivity $(\kappa)$ data [84] and model results for the EC-DMC-DEC-EP (1:1:1:1, vol.)$\mathrm{LiPF}_{6}$ system at $1.0 \mathrm{M}$ salt and various temperatures. Lab data (dark circles) is compared to model results (solid curves). Good fidelity of model predictions is seen over the entire temperature range of -60 to $25^{\circ} \mathrm{C}$.

Fig. 16: Contributions to electrolyte conductivity, considering the cation $\mathrm{Li}^{+}$. The system FECGBL-EMC-MB (2:2:3:3, mass)-LiFSI is used as a case study. Some factors from Eq. (6) have been normalized to evaluate their relative influence.

Fig. 17: Comparison of conductivity at $1 \mathrm{M}$ salt for Li-ion vs Na-ion systems, using $\mathrm{LiPF}_{6}$ and $\mathrm{NaPF}_{6}$ as the basis. In all but one case (EC-DMC-LiPF 6 ) the Na-based electrolytes yield higher conductivities than their lithium counterparts.

Fig. 18: LSS results for the ternary solvent system XYZ (proprietary)-GBL-EMC-LiPF 6 , showing optimized solvent proportions based on conductivity $(\kappa)$. Optimized proportions vary over temperature and salt concentration, allowing fine tuning of electrolyte formulation according to the conditions of the actual application. 


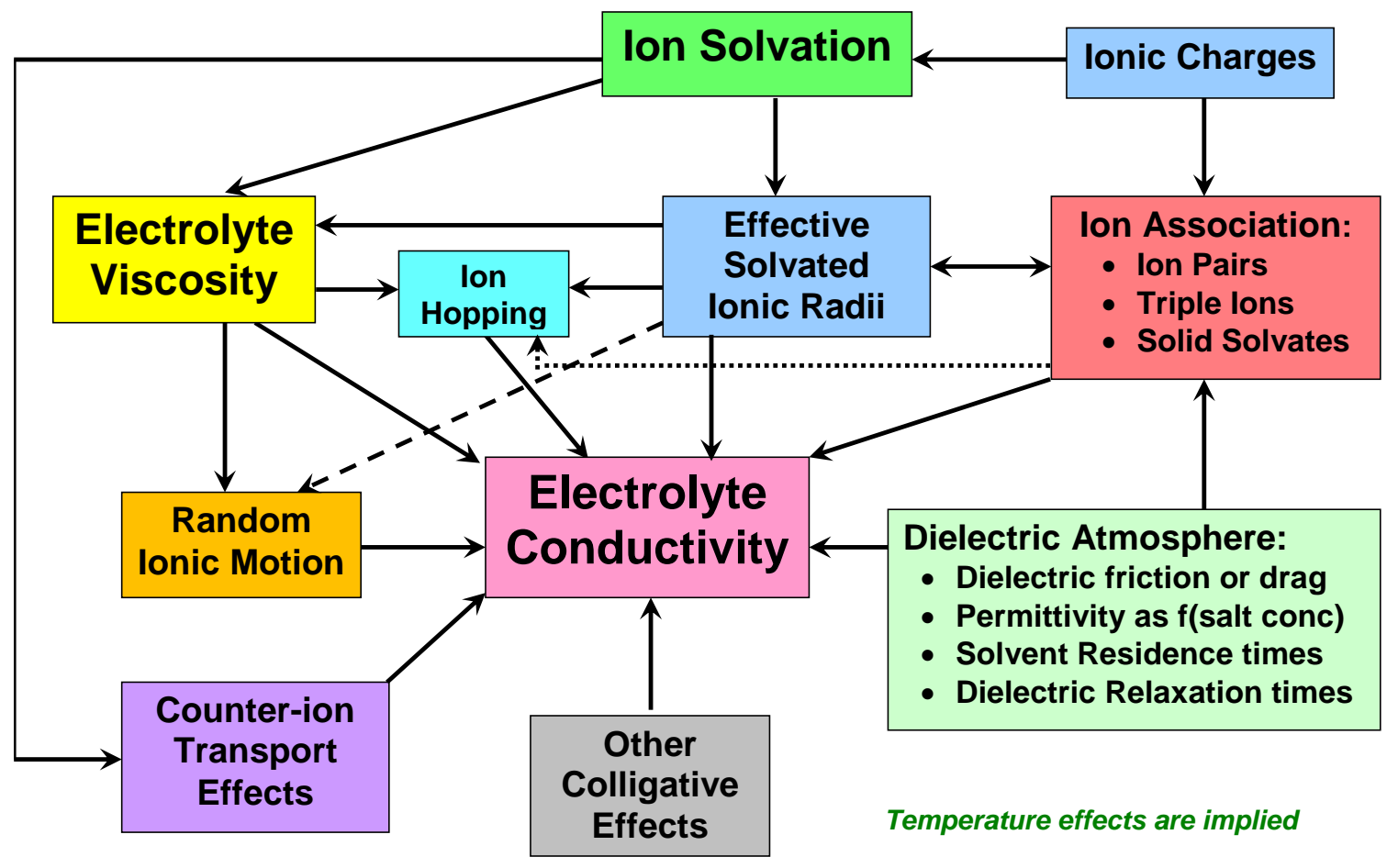

Fig. 1: Relationship of contributing factors to ionic conductivity, as incorporated into the AEM modeling approach. Ion solvation is a foundational attribute that underlies many aspects of electrolyte conductivity. 


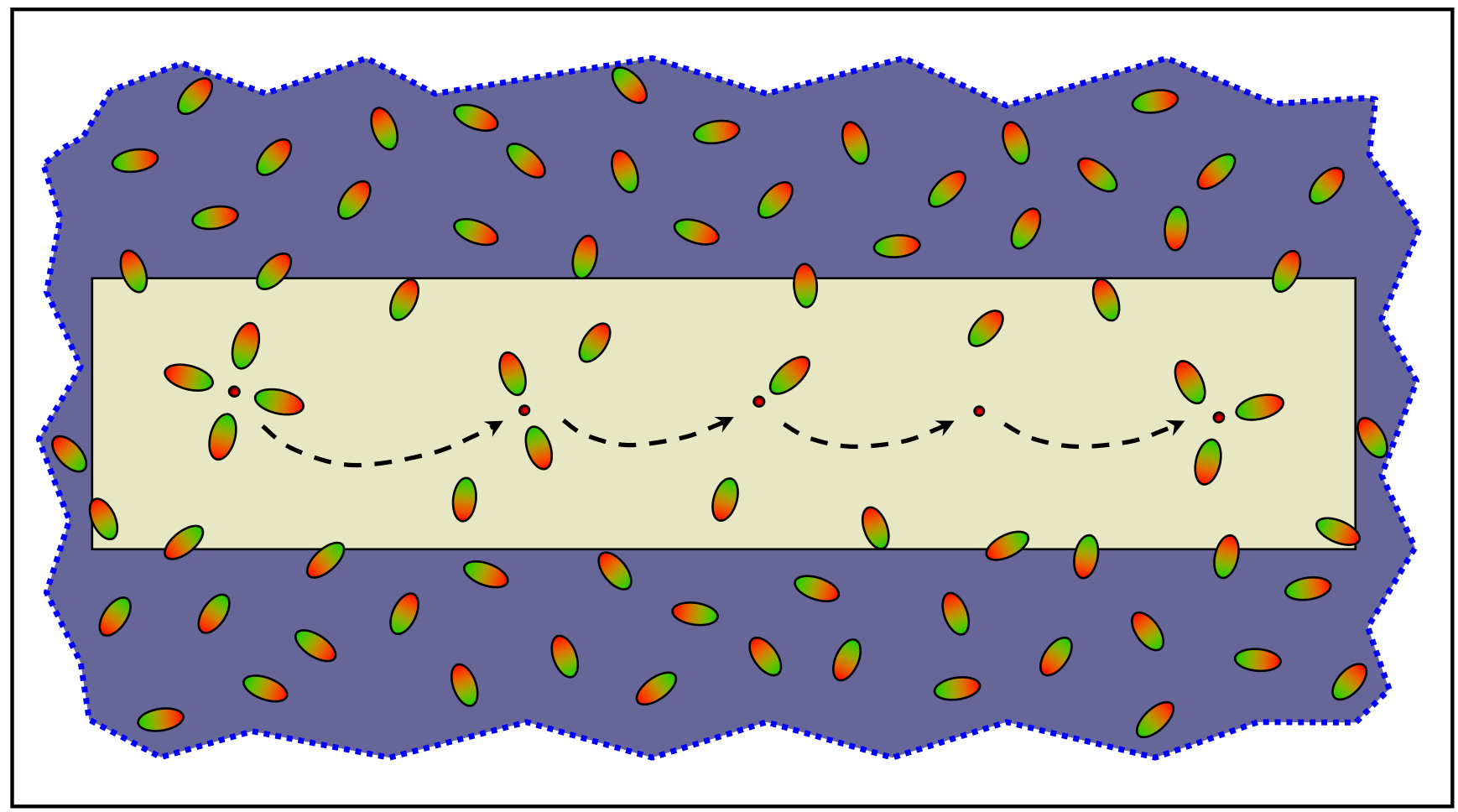

Fig. 2: Conceptual rendering of ion hopping in a liquid electrolyte system. Various solvation microstates are shown as the ion moves from left to right under Faradaic transport. 


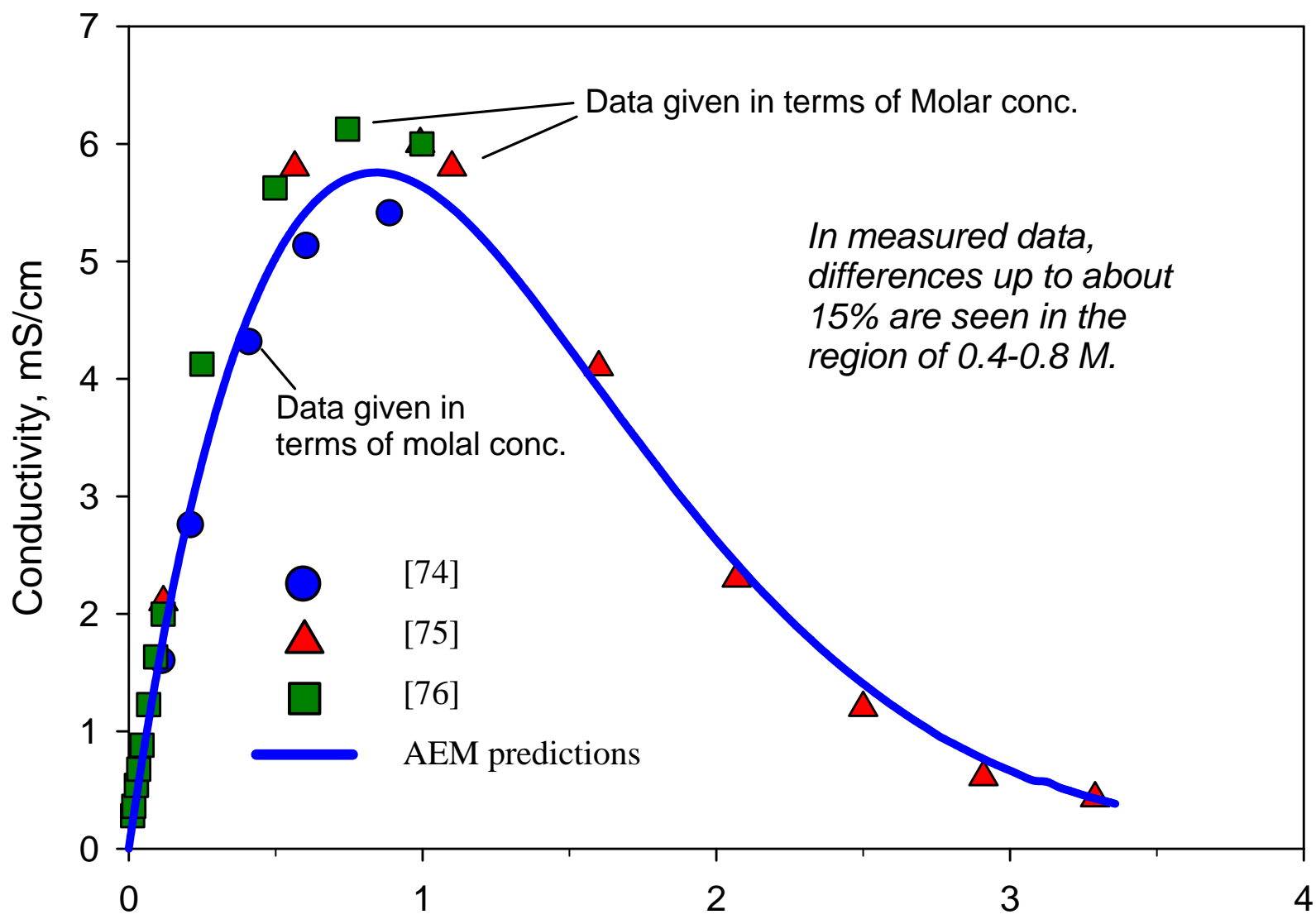

Salt Conc., Molar or molality

Fig. 3: Conductivity data and model results for the $\mathrm{PC}-\mathrm{LiPF}_{6}$ system at $25^{\circ} \mathrm{C}$ as a function of salt concentration. Some non-trivial discrepancies are seen between the lab data [74-76]. AEM gives good fidelity overall, even past 3M salt. 


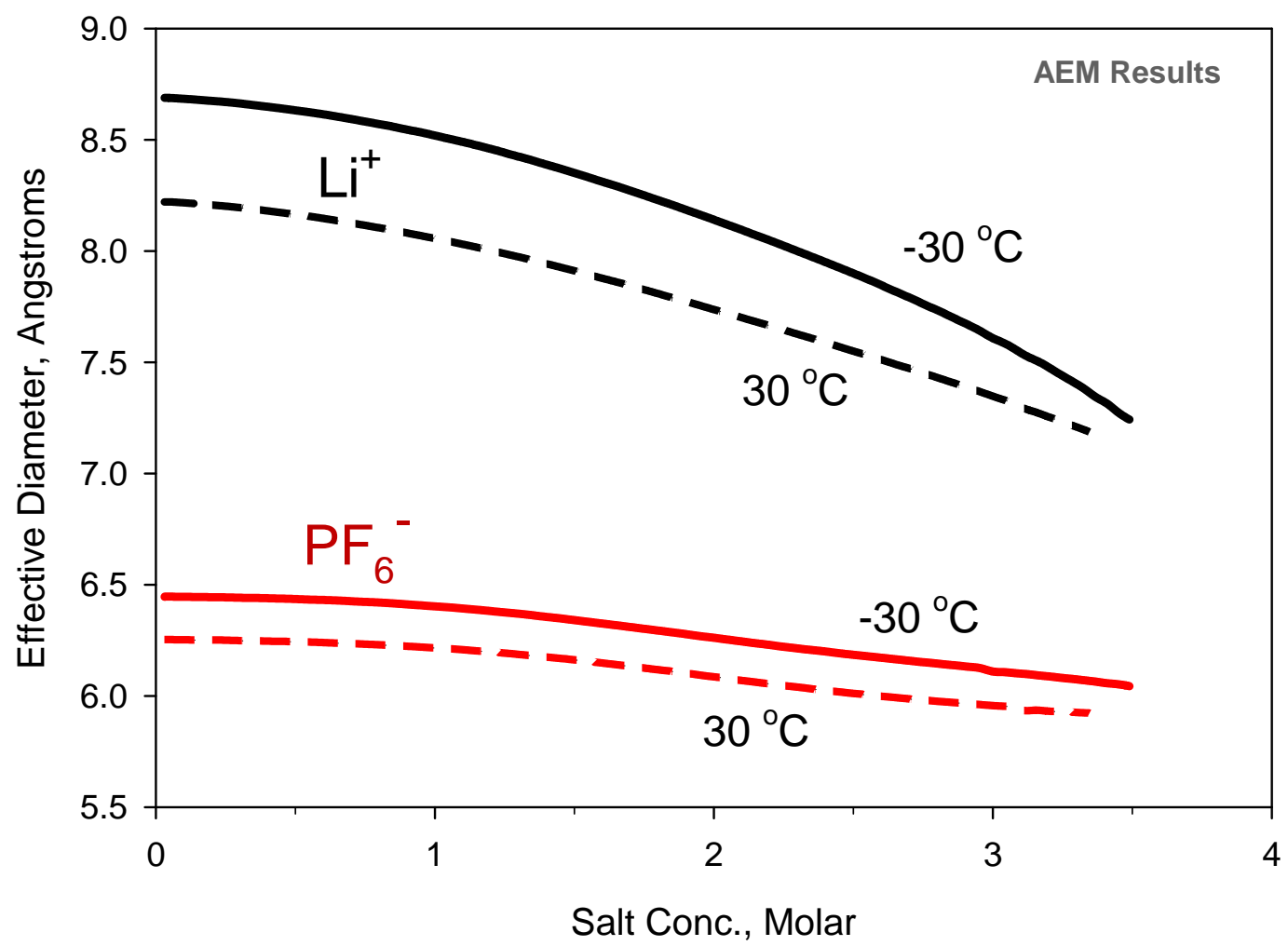

Fig. 4: Effective (transport) solvated ion sizes determined by the AEM for the PC$\mathrm{LiPF}_{6}$ systems at -30 and $30{ }^{\circ} \mathrm{C}$. Solvated cation and anion sizes are seen to decrease with increasing salt concentration and with increasing temperature. In this system, solvated anion sizes are less than their cation counterparts. 

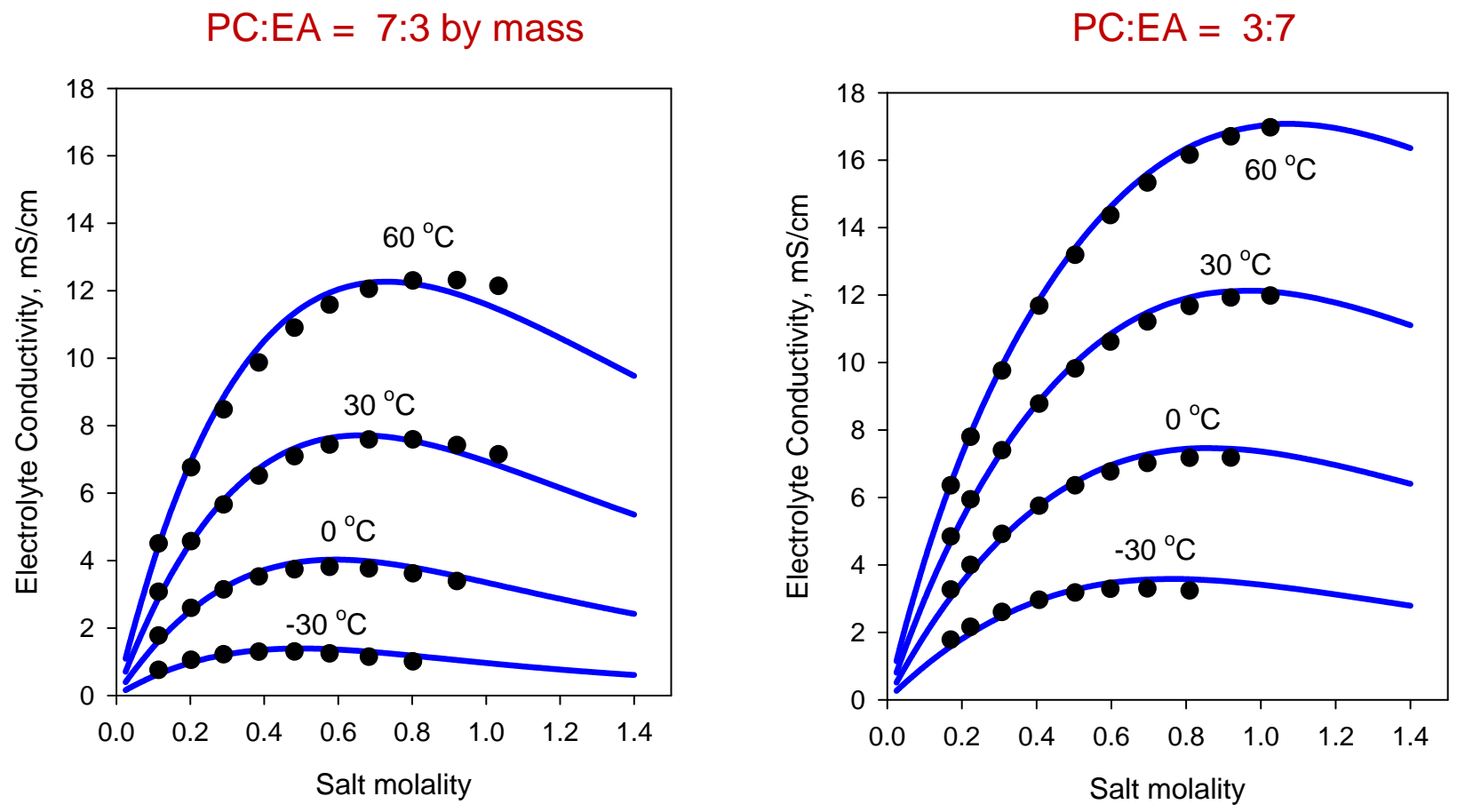

Fig. 5: Conductivity data and model results for the PC-EA-LiBOB system at various temperatures and as a function of salt concentration. Lab data (dark circles, [77]) is compared to model results (solid curves). 


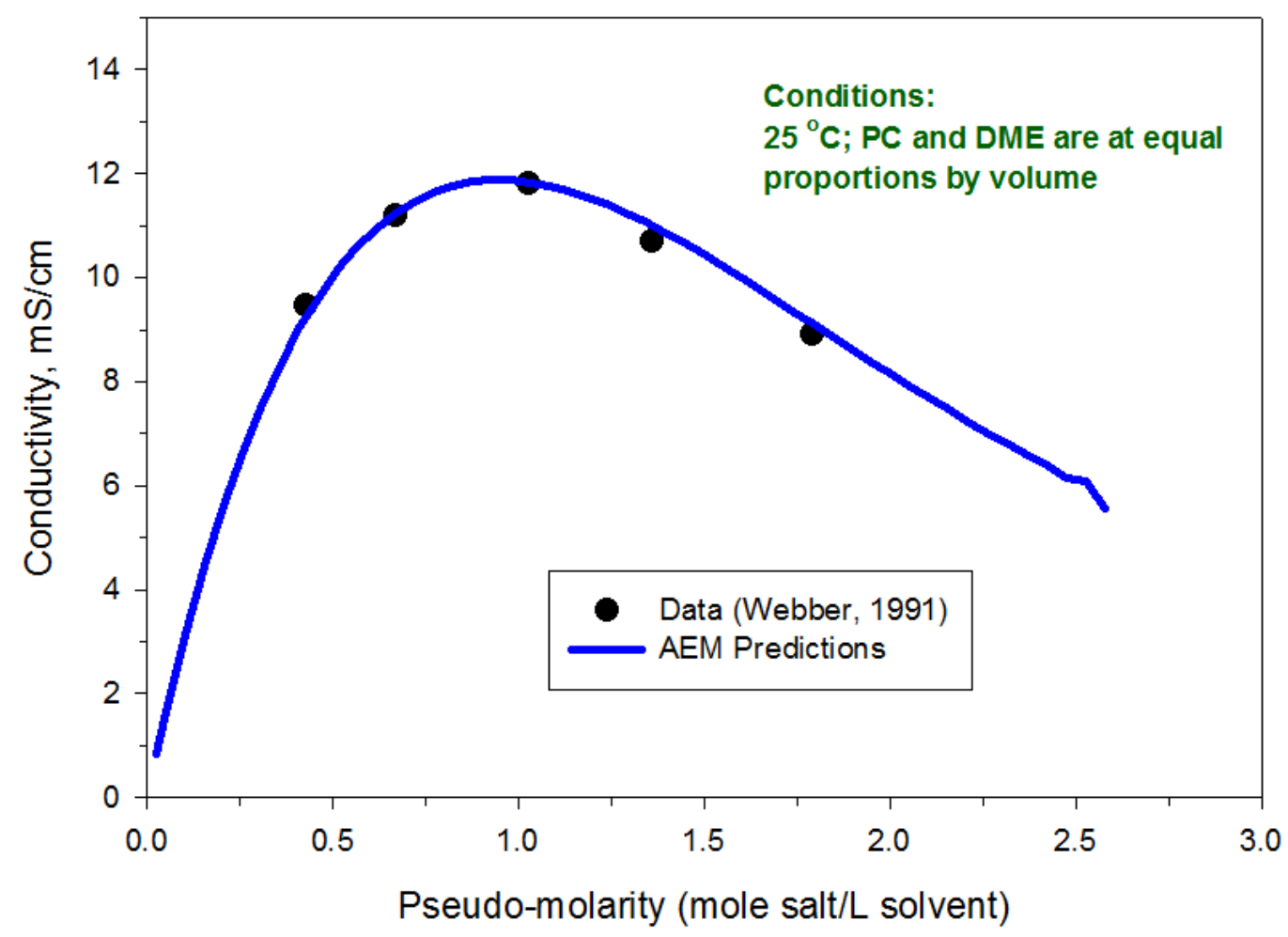

Fig. 6: Conductivity data and model results for the PC-DME-LiTFSI system at $25^{\circ} \mathrm{C}$ as a function of salt concentration. Lab data (dark circles, [78]) is compared to model results (solid curve). The slight bend in the curve at around 2.5 pseudo-molarity is due to model adjustments made in response to a critical packing fraction reached at that condition. 

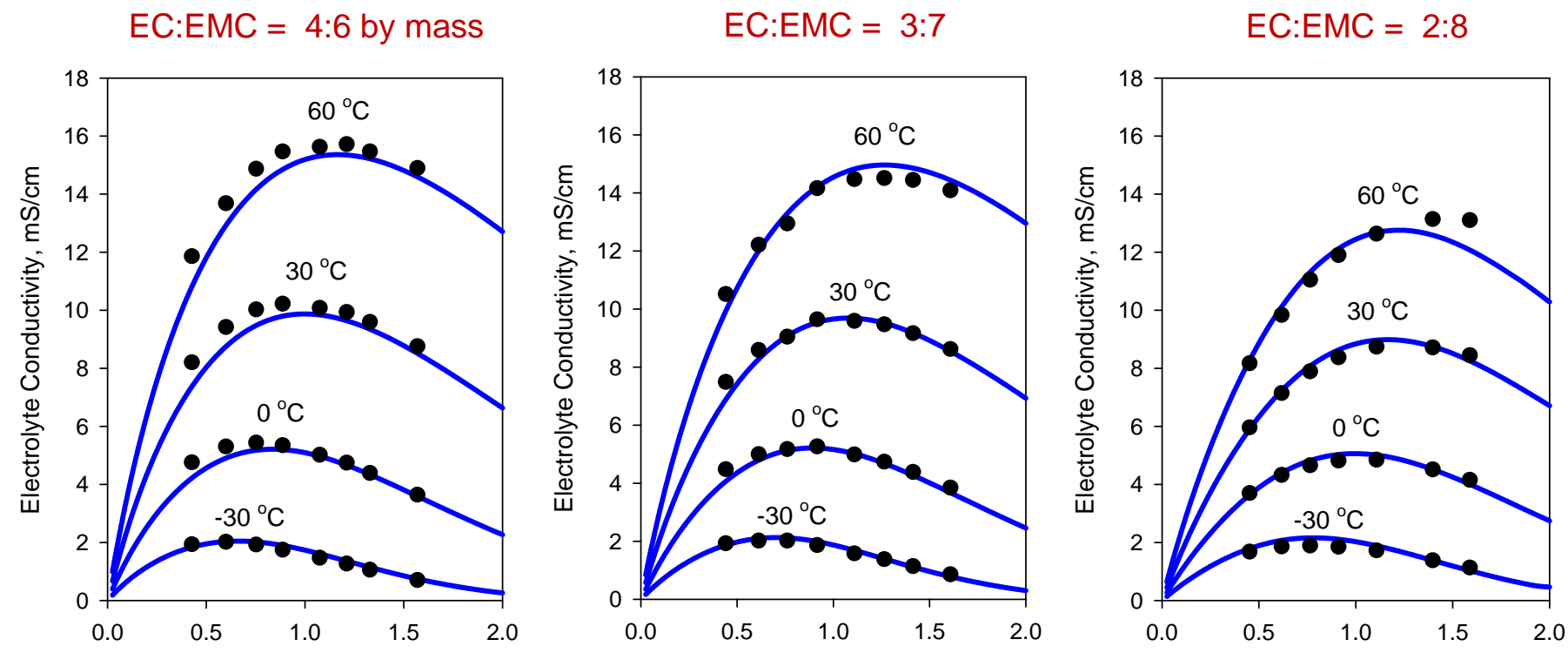

--- Salt Conc., molality ---

Fig. 7: Conductivity data and model results for the EC-EMC-LiPF 6 system at various temperatures and as a function of salt concentration. Lab data (dark circles, [79]) is compared to model results (solid curves). Overall average deviation between model and lab data is $5 \%$. 


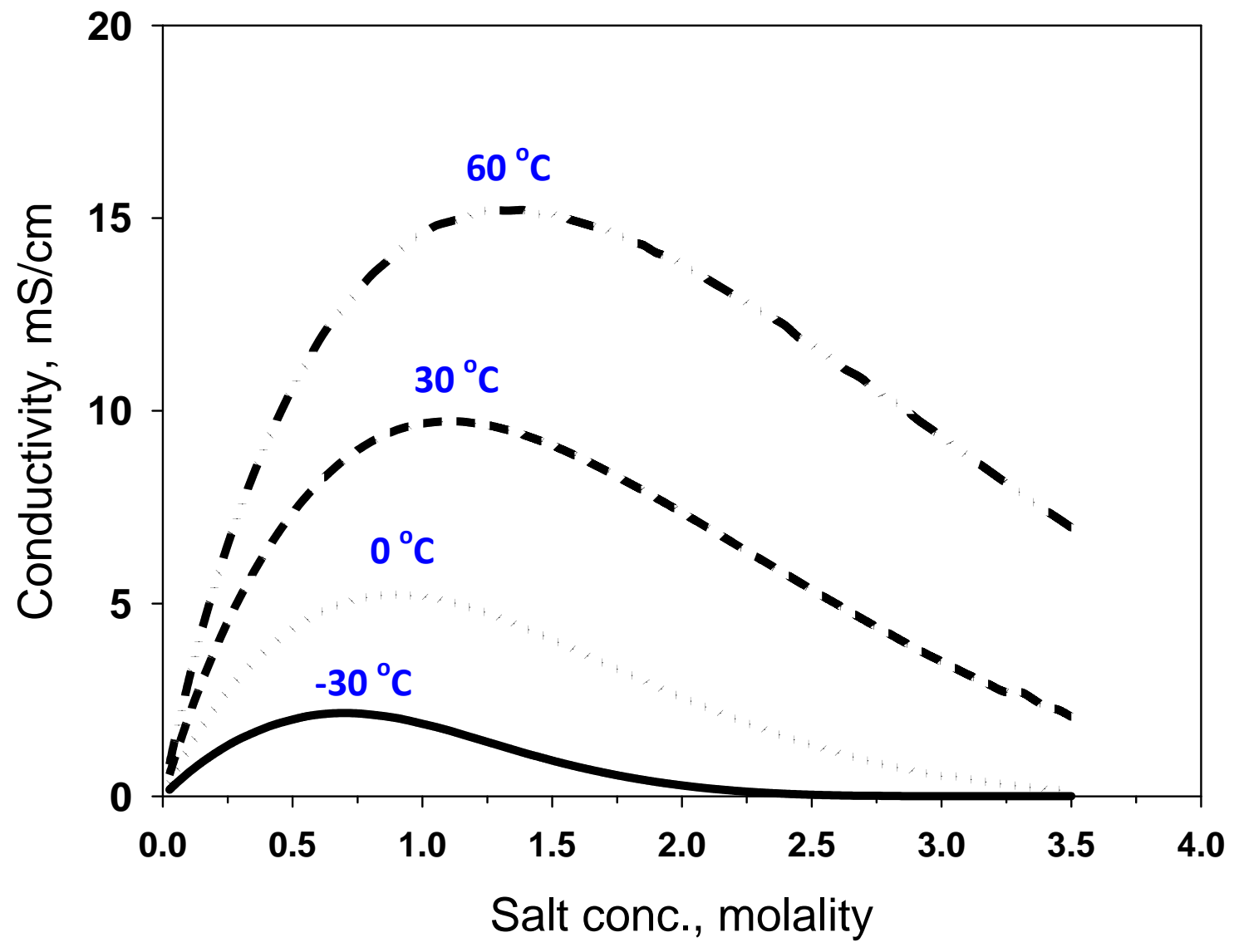

Fig. 8: AEM results for the EC-EMC-LiPF 6 system at various temperatures and as a function of extended salt concentration. The very slight bend in the curves at higher molality is due to model adjustments made in response to a critical packing fraction reached at those conditions, which is a function of temperature. 


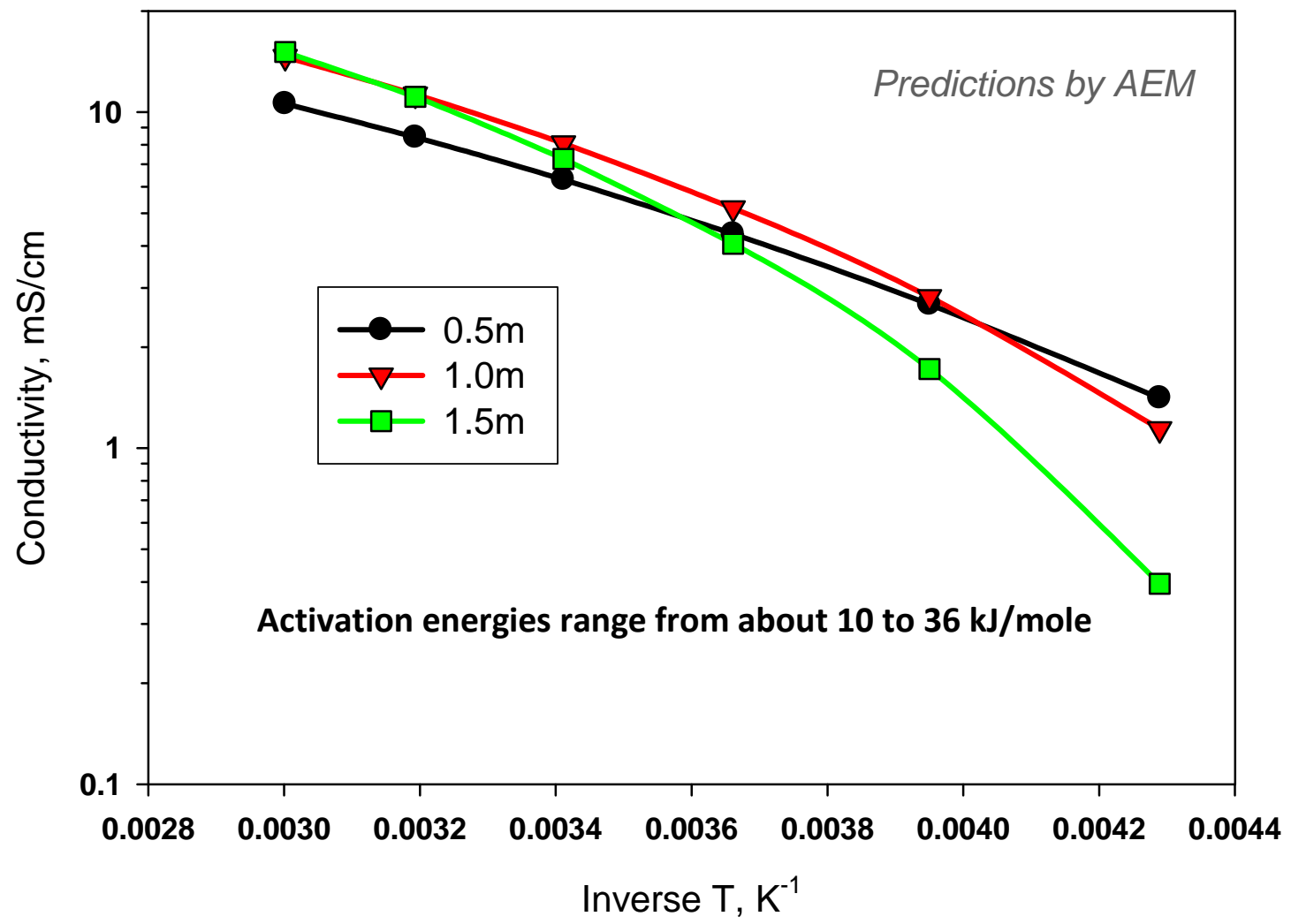

Fig. 9: Arrhenius analyses of conductivity based on AEM results for the EC-EMC-LiPF 6 system, using salt concentration (molality) as the parameter. Activation energies based on conductivity increase at lower temperatures and higher salt concentrations. This has implications for polarized Li-ion cells operating at low temperatures. 

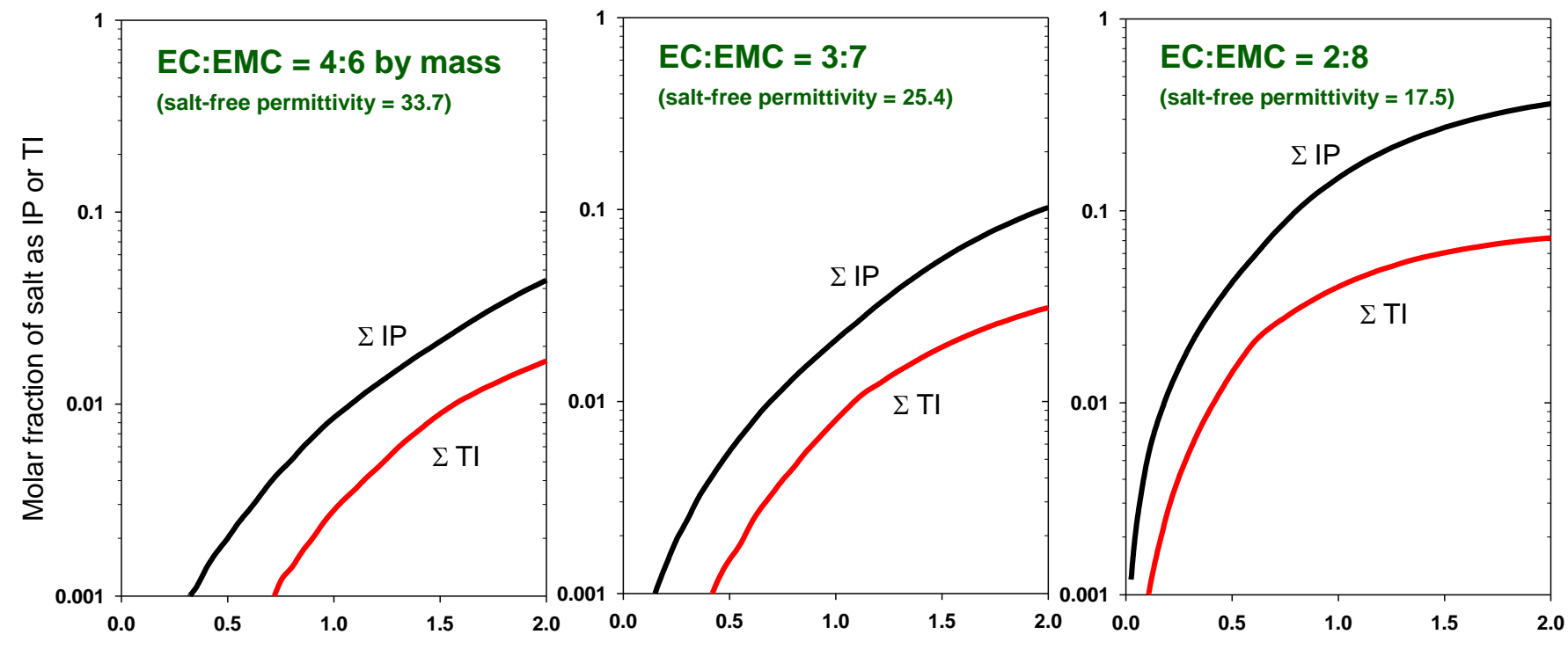

--- Salt Conc., molality ---

Fig. 10: Prediction of ion association species for the EC-EMC-LiPF 6 system at $30{ }^{\circ} \mathrm{C}$, as functions of solvent composition and salt concentration. The sum of ion pairs $\left(\sum I P\right)$ is comprised of contact ion pairs, solvent-separated ion pairs and solvent-shared ion pairs, while the triple ion summation $\left(\sum \mathrm{TI}\right)$ is comprised of $\mathrm{ABA}^{+}$and $\mathrm{BAB}^{-}$species. 


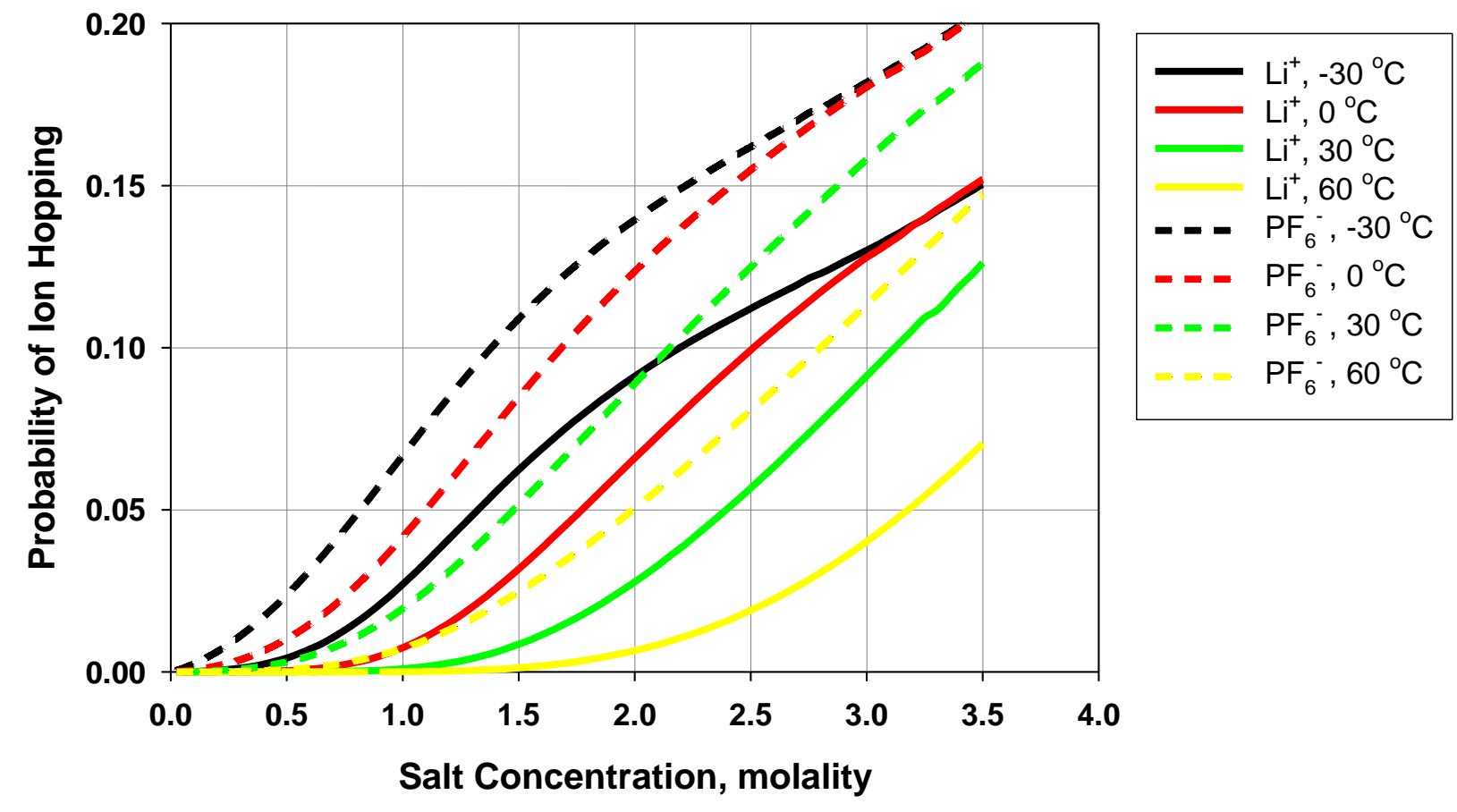

Fig. 11: Probability of ion hopping for $\mathrm{Li}^{+}$and $\mathrm{PF}_{6}{ }^{-}$in EC-EMC. Ion hopping is more prevalent for lesser-solvated ions (here, anions) and at lower temperatures. 


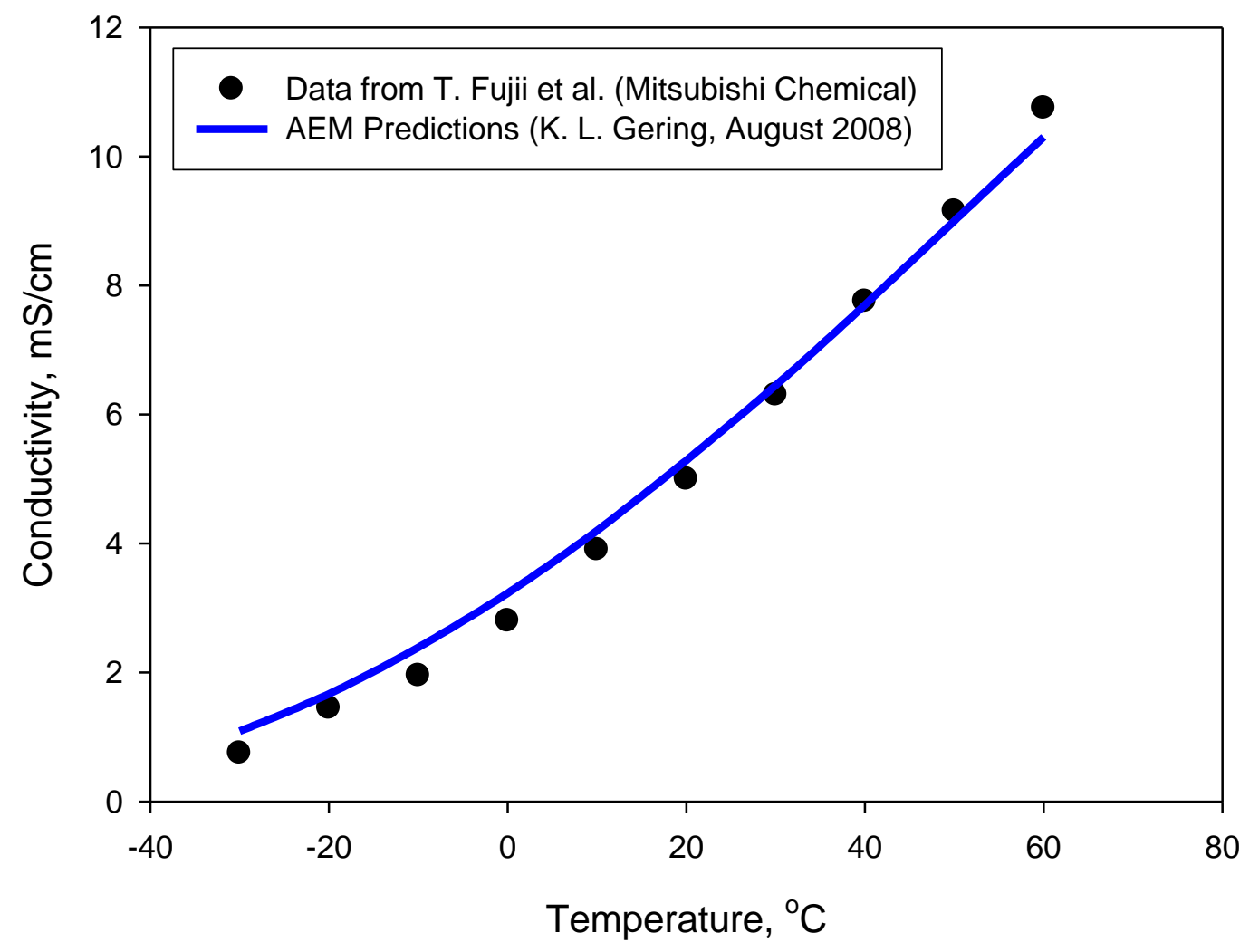

Fig. 12: Conductivity data [81] and model results for the EC-EMC-LiBOB system at various temperatures and at $0.85 \mathrm{M}$ salt. Lab data (dark circles) is compared to model results (solid curve). 


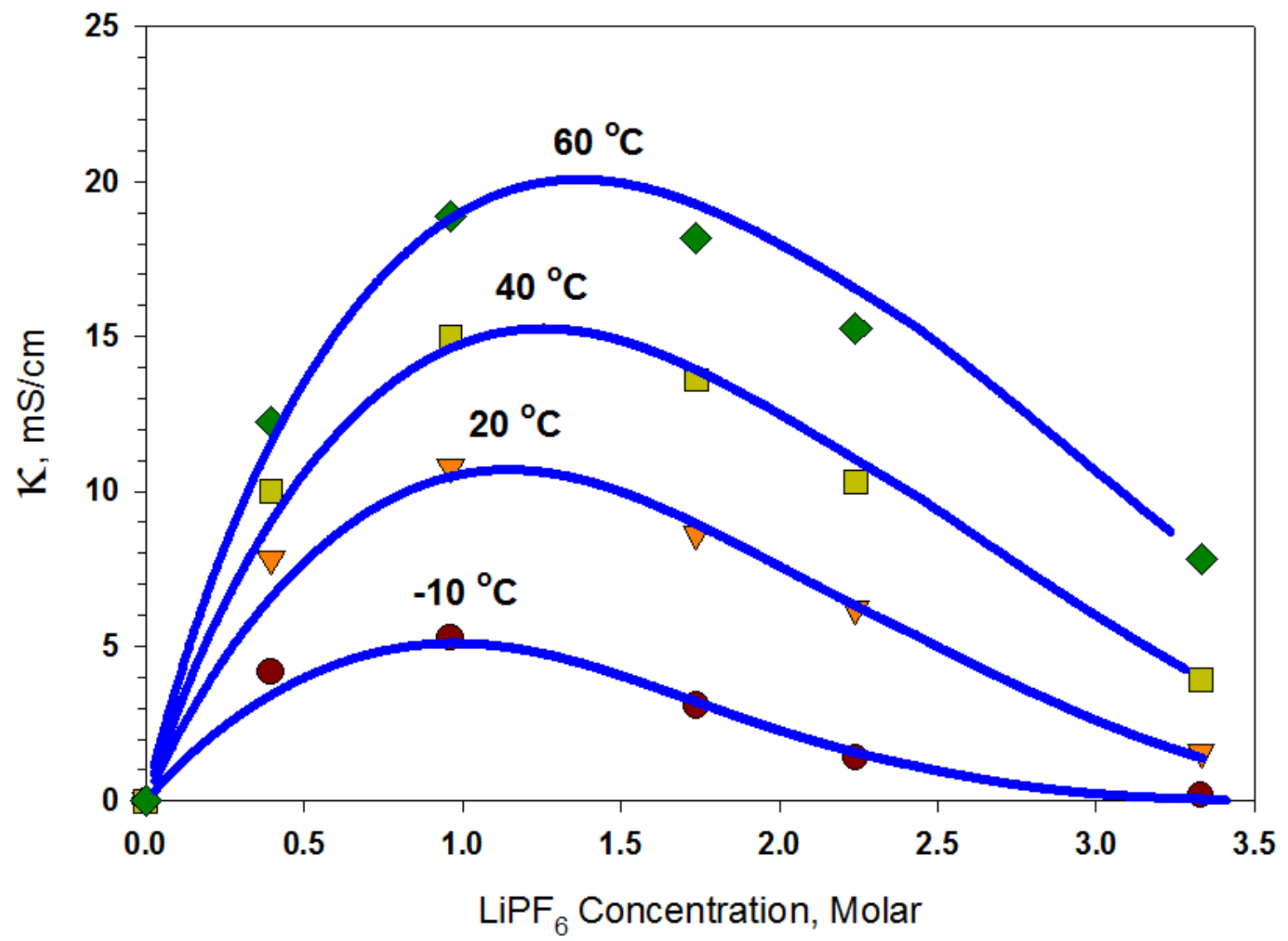

Fig. 13: Conductivity ( $\kappa)$ data [82] and model results for the EC-PC-DMC (27:10:63, vol.)$\mathrm{LiPF}_{6}$ system at various temperatures and over salt concentration. Lab data (symbols) is compared to model results (solid curves). 

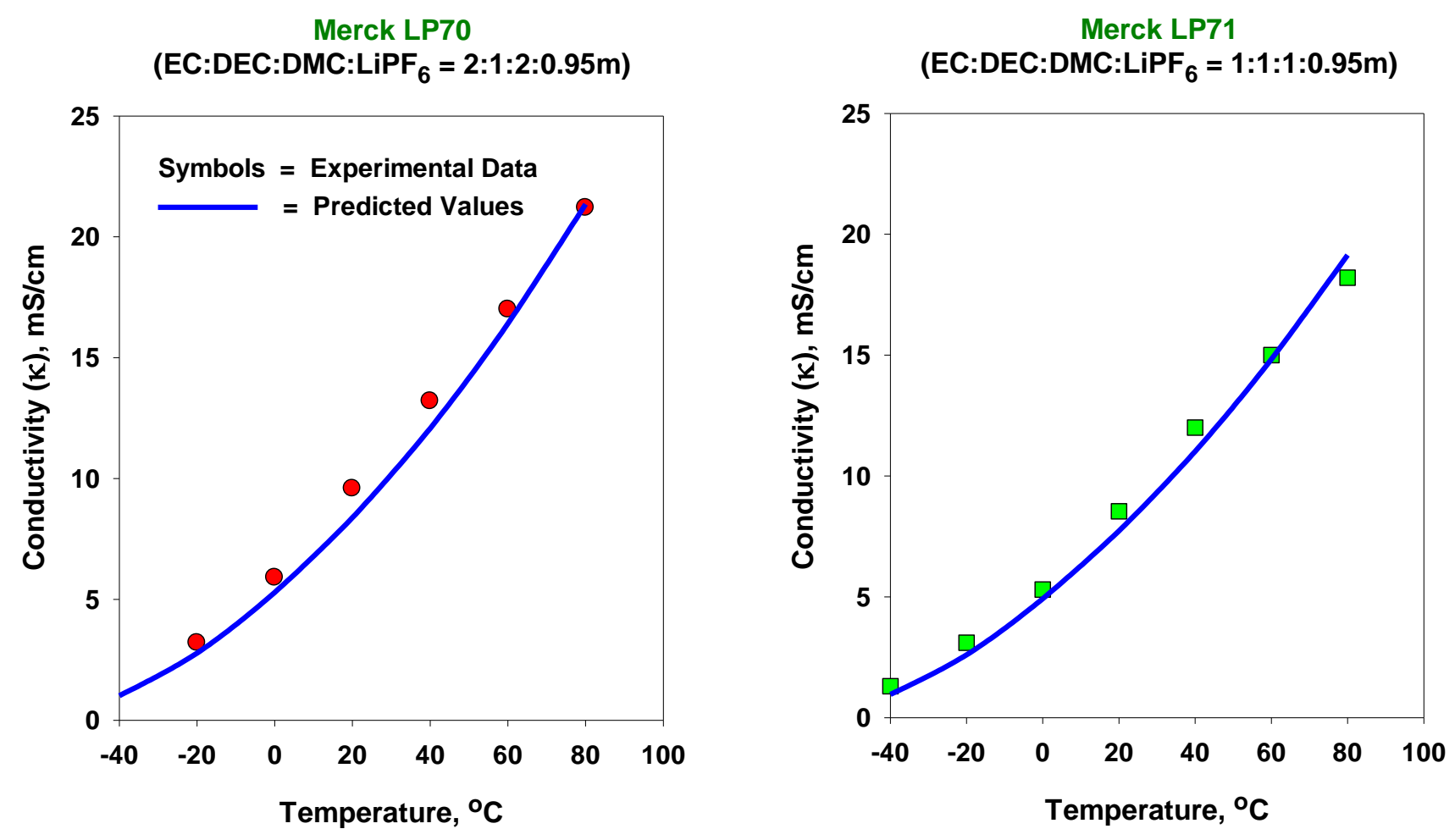

Fig. 14: Conductivity data [83] and model results for the EC-DEC-DMC-LiPF 6 system at 0.95 molality salt, two sets of solvent proportions and various temperatures. Lab data (dark circles) is compared to model results (solid curves). Good fidelity of model predictions is seen over the entire temperature range of -40 to $80{ }^{\circ} \mathrm{C}$. 


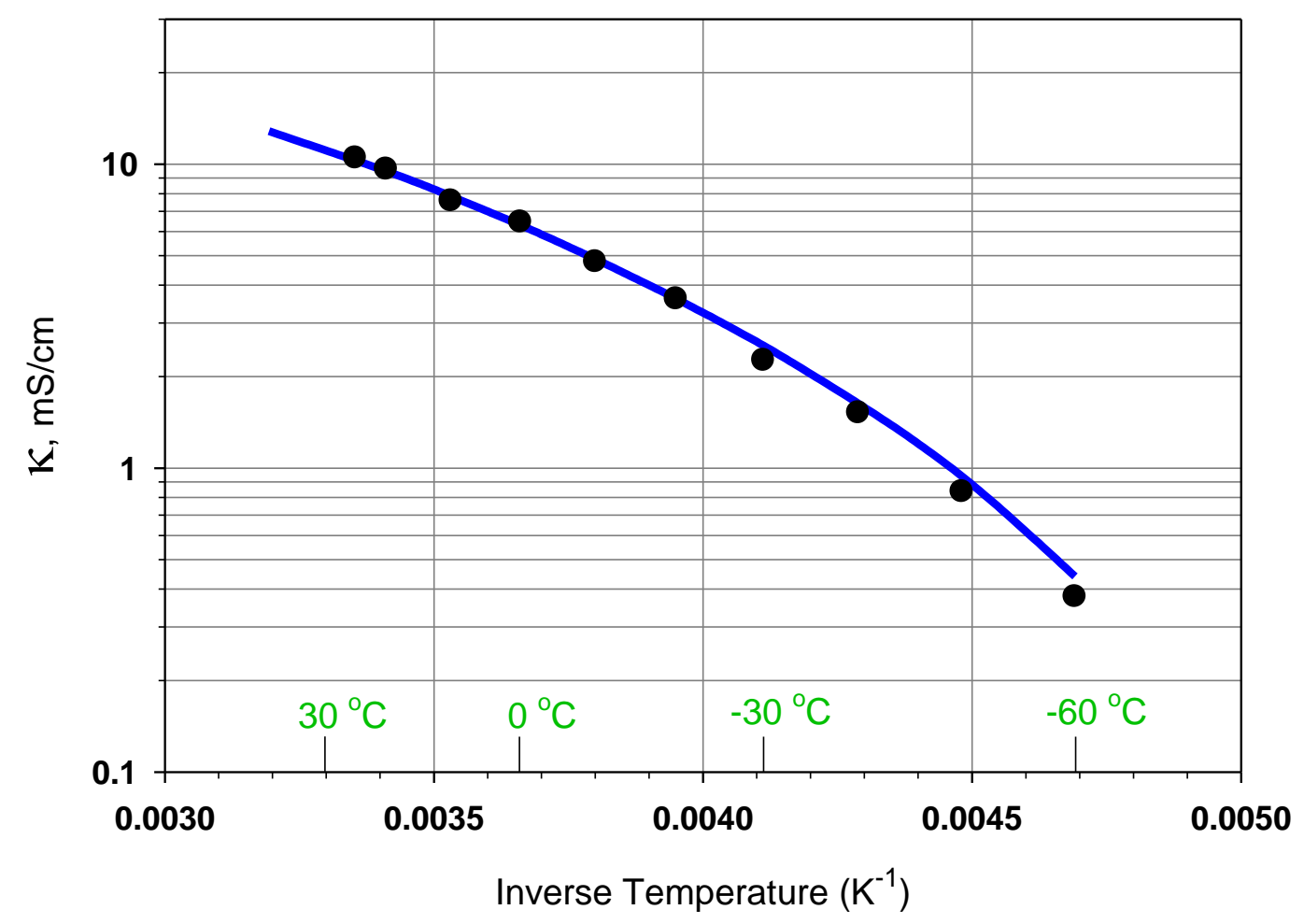

Fig. 15: Conductivity ( $\kappa)$ data [84] and model results for the EC-DMC-DEC-EP (1:1:1:1, vol.)$\mathrm{LiPF}_{6}$ system at $1.0 \mathrm{M}$ salt and various temperatures. Lab data (dark circles) is compared to model results (solid curves). Good fidelity of model predictions is seen over the entire temperature range of -60 to $25{ }^{\circ} \mathrm{C}$. 


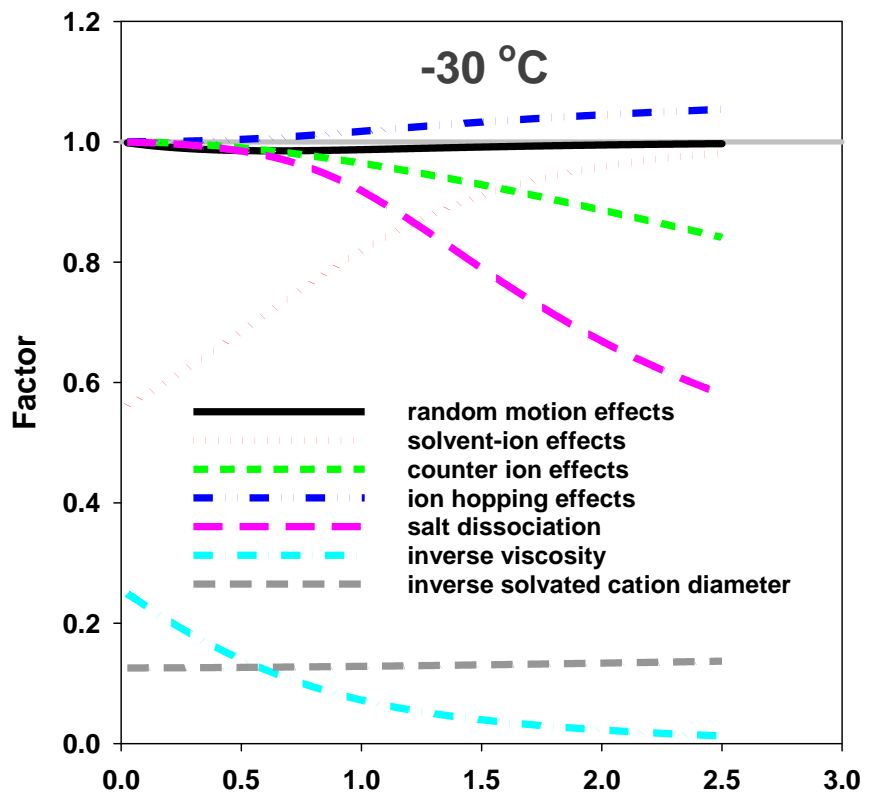

Salt Conc., molality

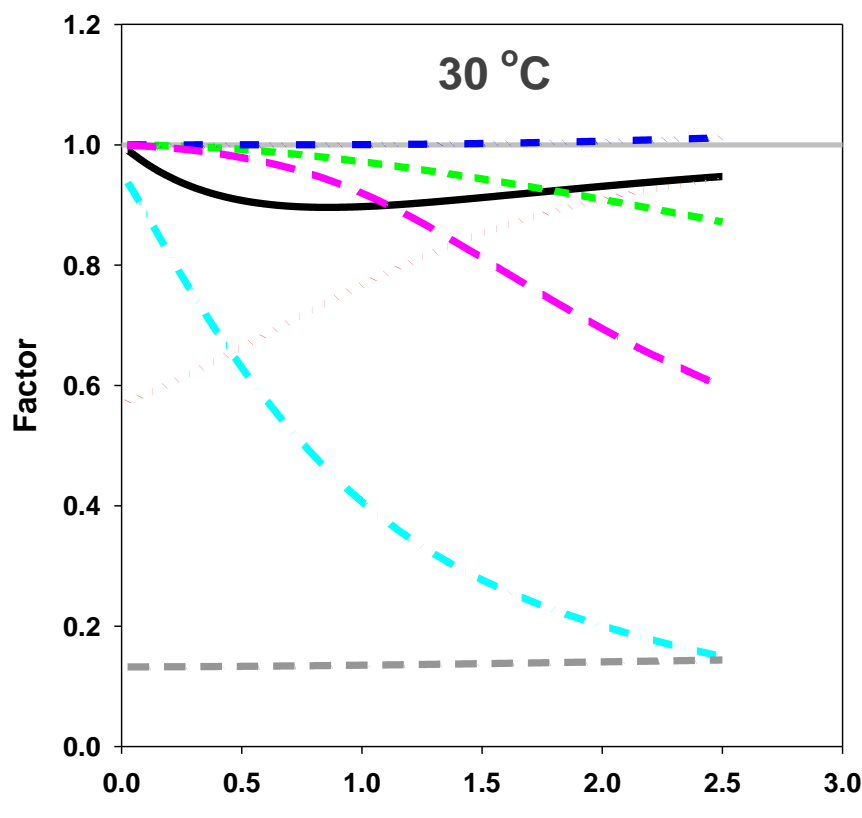

Salt Conc., molality

Fig. 16: Contributions to electrolyte conductivity, considering the cation $\mathrm{Li}^{+}$. The system FEC-GBL-EMC-MB (2:2:3:3, mass)-LiFSI is used as a case study. Some factors from Eq. (6) have been normalized to evaluate their relative influence. 


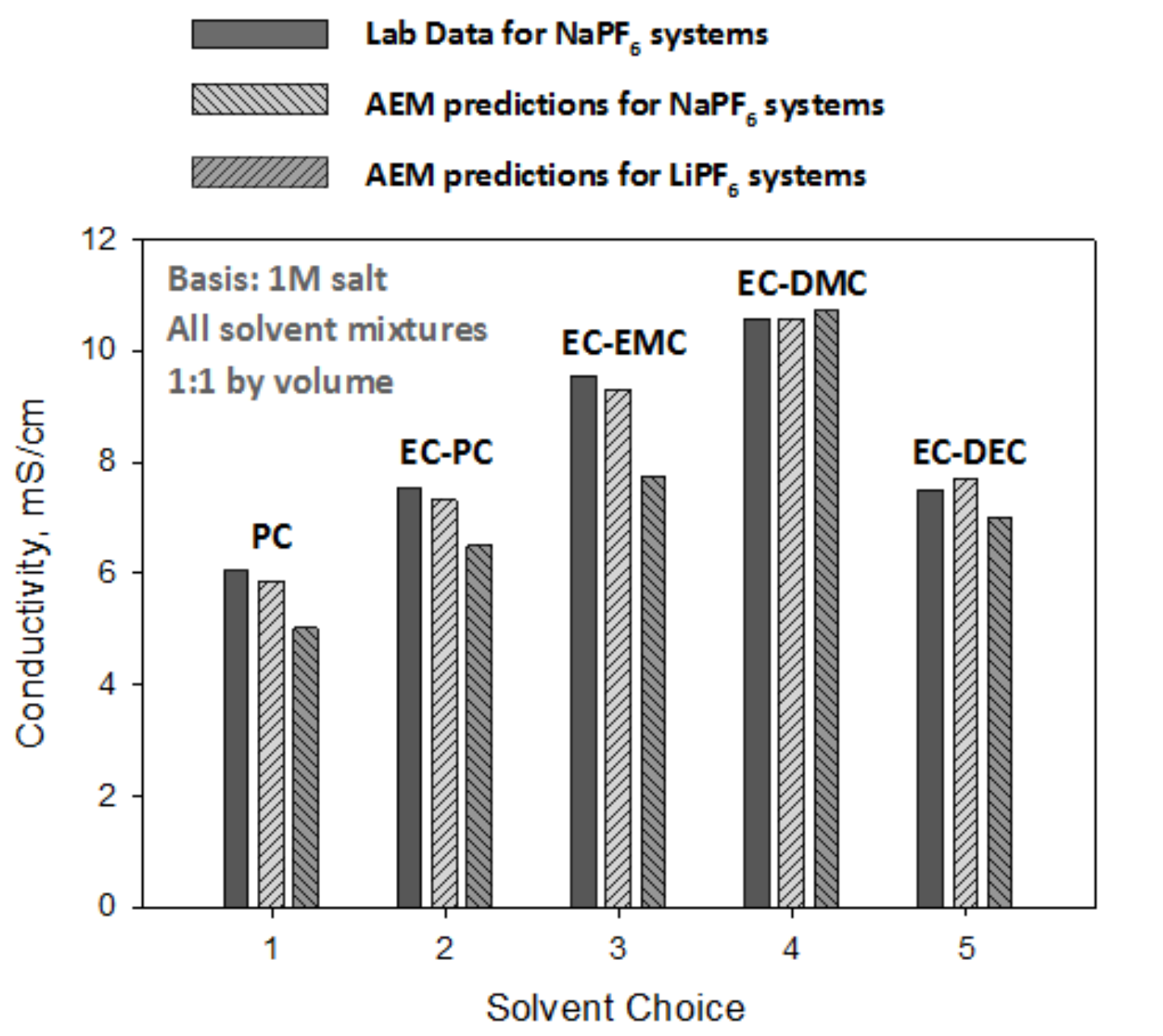

Fig. 17: Comparison of conductivity at $1 \mathrm{M}$ salt for Li-ion vs Na-ion systems, using $\mathrm{LiPF}_{6}$ and $\mathrm{NaPF}_{6}$ as the basis. Lab temperatures are $21{ }^{\circ} \mathrm{C} \pm 1{ }^{\circ} \mathrm{C}$, and model temperature is $20{ }^{\circ} \mathrm{C}$. In all but one case $\left(\mathrm{EC}-\mathrm{DMC}-\mathrm{LiPF}_{6}\right)$ the Na-based electrolytes yield higher conductivities than their lithium counterparts. 

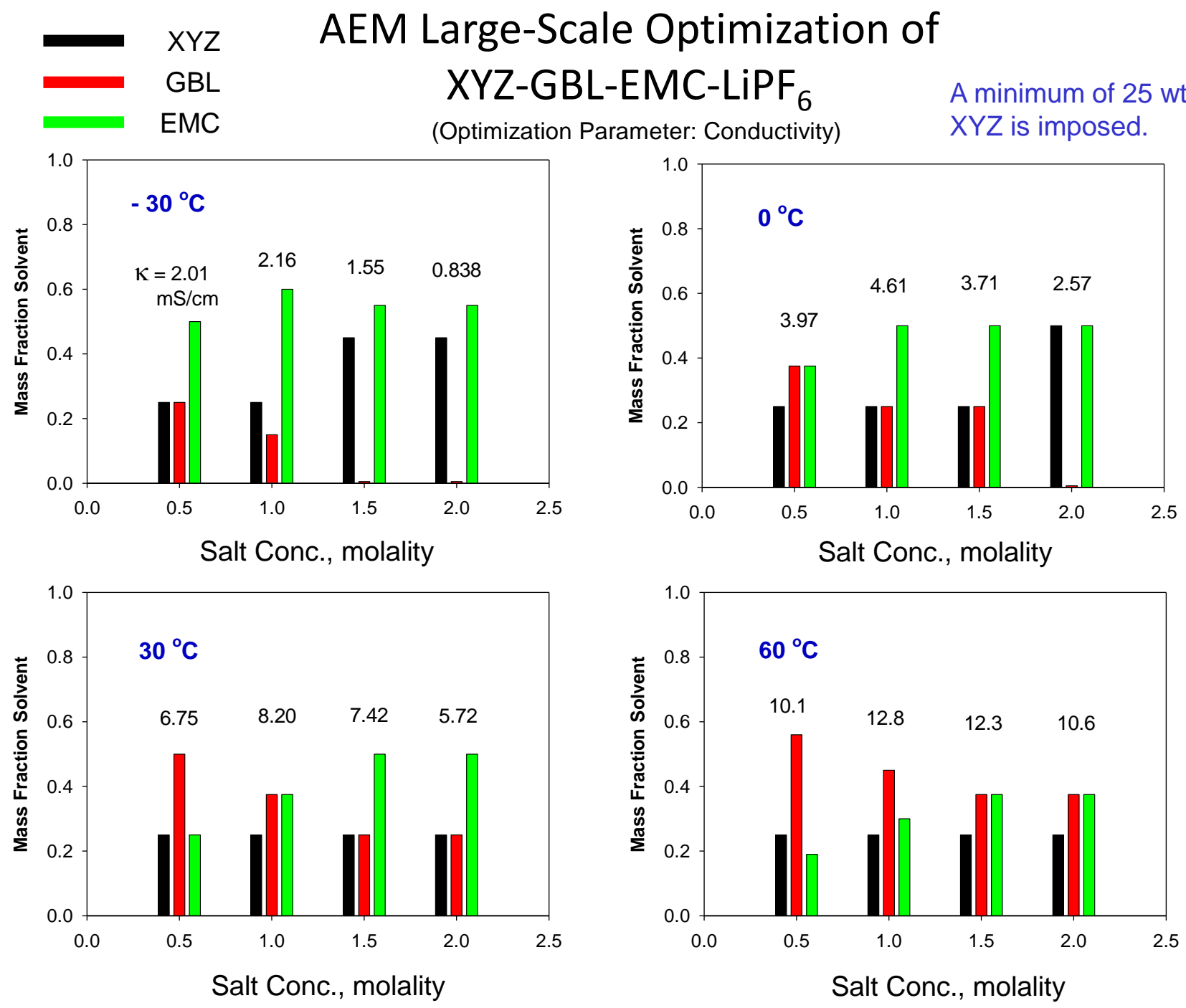

Fig. 18: LSS results for the ternary solvent system XYZ (proprietary)-GBL-EMC-LiPF, showing optimized solvent proportions based on conductivity $(\kappa)$. Optimized proportions vary over temperature and salt concentration, allowing fine tuning of electrolyte formulation according to the conditions of the actual application. 

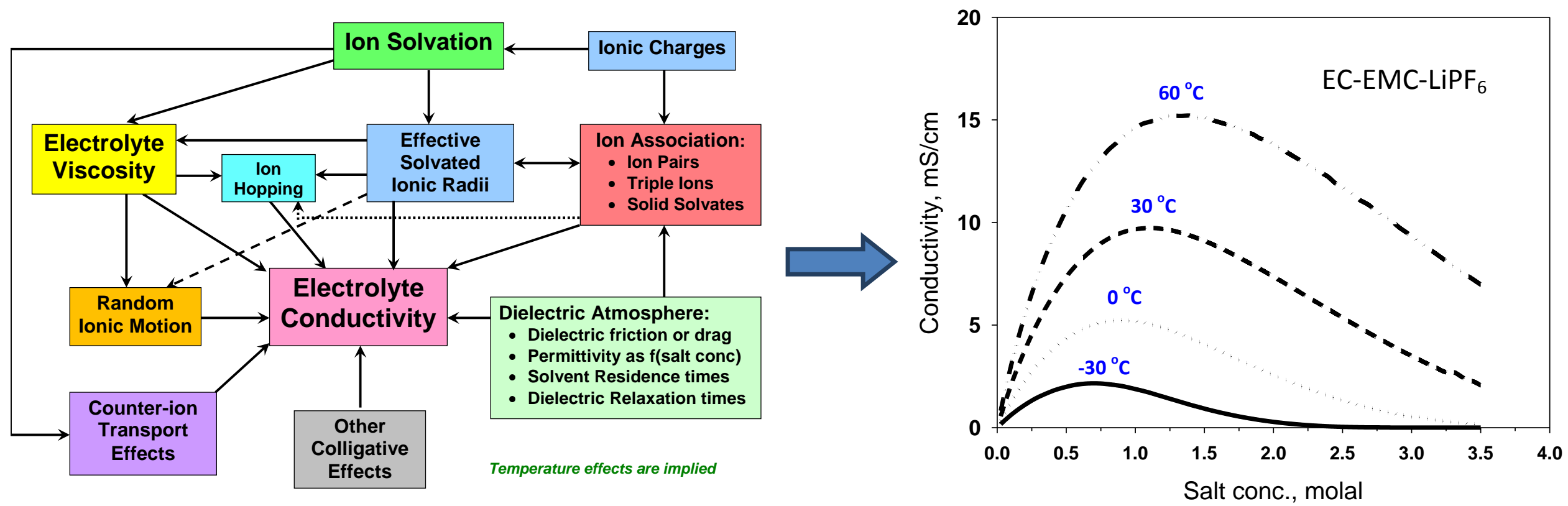